\title{
A 3-D model for ocean waves over a Columb-damping poroelastic seabed
}

\author{
M. Lin ${ }^{\mathrm{a}}$, D.-S. Jeng ${ }^{\mathrm{b}, *, 1}$ \\ ${ }^{a}$ Institute of Mechanics, Chinese Academy of Science, Beijing 1008, China \\ b School of Engineering, Griffith University Gold Coast Campus, QLD 9726, Australia
}

Received 12 June 2003; accepted 7 October 2003

\begin{abstract}
The evaluation of the wave-induced seabed instability in the vicinity of a breakwater is particularly important for coastal and geotechnical engineers involved in the design of coastal structures. In this paper, an analytical solution for three-dimensional short-crested wave-induced seabed instability in a Coulomb-damping porous seabed is derived. The partial wave reflection and self-weight of breakwater are also considered in the new solution. Based on the analytical solution, we examine (1) the wave-induced soil response at different location; (2) the maximum liquefaction and shear failure depth in coarse and fine sand; (3) the effects of reflection coefficients; and (4) the added stresses due to the self-weight of the breakwater.
\end{abstract}

(C) 2003 Elsevier Ltd. All rights reserved.

Keywords: Liquefaction; Shear failure; Scour; Wave loading; Seabed response

\section{Introduction}

Protection of the coastal environment is vital for countries like Australia, where $86 \%$ of the total population is concentrated around the coasts. Marine structures such as caissons and seawalls are commonly adopted for such protection. Although the protection of marine structures has been extensively studied in recent years, understanding of their interaction with waves and the seabed is far from complete. Damage of marine structures still occurs from time to time, with two general failure

* Corresponding author. Tel.: +61-7-5552-8683; fax: +61-7-5552-8065.

E-mail addresses: d.jeng@griffith.edu.au; dsjeng@hotmail.com (D.-S. Jeng).

${ }^{1}$ New address: Department of Civil Engineering, The University of Sydney, NSW 2006, Australia. 
modes evident. The first mode is that of structural failure, caused by wave forces acting on and damaging the structure itself. The second mode is that of foundation failure, caused by liquefaction or erosion of the seabed in the vicinity of the structure, resulting in collapse of the structure as a whole. This study will focus on the mechanism of wave-induced seabed instability in the vicinity of a breakwater.

The wave phenomenon in the vicinity of a breakwater or seawall is complicated, which normally involves with wave interactions and wave-structure interactions. The simplest case is a fully reflection in the direction normal to the wall (i.e. twodimensional standing waves), which has been widely studied in the past (Irie and Ndaoka, 1984). In fact, the wave interactions in the vicinity of a breakwater is a three-dimensional short-crested waves, which could be an interaction of two or more wave trains. This three-dimensional wave system has been studied by numerous researchers (Fuchs, 1952; Silvester, 1972; Hsu et al., 1979; Jeng, 2002).

In general, the wave-induced seabed instability in the vicinity of a breakwater can be classified into three categories: scour, liquefaction and shear failure (Sumer and Fredsoe, 2000). Among these, scouring has been studied by coastal engineers, while liquefaction and shear failure have been studied by geotechnical engineers in the past. The phenomenon of scouring around a coastal structure has been extensively studied in the past, and numerous empirical formulas have been proposed in the past (Irie and Ndaoka, 1984; Xie, 1983; Lin et al., 1986; Jan and Lin, 1998). Basically, the scouring depth is related to the mass transportation and velocity distribution (Tanaka et al., 1972; Carter et al., 1973; Hsu et al., 1980; Hsu and Silvester, 1989; Gao and Zhao, 1995; Sumer and Fredsoe, 2000), and the onset of scouring and scouring depth are two main concerns (Sumer and Fredsoe, 2000). Liquefaction occurs when the wave-induced excess pore pressure is large enough to overcome the self-weight of the soil particles, which is generally occurs in finer seabeds (Zen and Yamazaki, 1993; Jeng, 1997). Shear failure is caused by the wave-induced shear forces acting on the soil particles, which can be determined by Mohr-Columbia criteria (Griffith, 1986). Basically, liquefaction is a kind of vertical movement of the soil particles, while shear failure is a horizontal movement. In this study, we will focus on the wave-induced liquefaction and shear failure in the vicinity of a breakwater.

Since the estimation of the wave-induced liquefaction and shear failure is based on the wave-induced pore pressure and effective stresses within the seabed, the wave-induced soil response will be an important parameter. Most previous investigations for wave-seabed interaction have been based on quasi-static approach, i.e. the conventional consolidation equation (Yamamoto et al., 1978; Tsai, 1995; Jeng, 1997), in which the acceleration due to soil and pore fluid motions are excluded. Some research has considered a more advanced approach by considering the acceleration of soil motion such as $u-p$ approximation (Jeng et al., 1999; Jeng and Rahman, 2000; Jeng and Lee, 2001). A full dynamic soil behavior has also been considered recently (Jeng and Cha, 2003). Coulomb-damping friction, which is particularly important for sandy and clay seabeds, has also been considered in some models (Yamamoto, 1982; Lin, 2001; Lee et al., 2002). A comparison between various existing models for the wave-seabed-interaction has 
been performed in the authors' recent papers (Lin, 2003; Lin and Jeng, 2003). However, it is only limited to two-dimensional case and wave-induced soil response, neither three-dimensional cases nor wave-induced seabed instability.

In this paper, the previous work regarding Columb-damping friction from twodimensional progressive waves to three-dimensional short-crested wave system (partially reflection). The self-weight of breakwater will also be considered in the new model. Based on the new solution, the wave-induced liquefaction and shear failure will be examined.

\section{Boundary value problem}

Consider that a progressive wave train on water of uniform depth $(d)$ obliquely approaches a rigid vertical wall and then partial reflect from it (see Fig. 1). The approaching and reflecting progressive waves are at an angle, resulting in an oblique standing wave (i.e. short-crested wave). This wave system has a surface elevation diamond-shaped crest pattern. A Cartesian coordinate system is assigned with the $x$-axis in the resultant direction of wave propagation that is parallel to the wall, the $y$-axis normal to the wall, while $z$-axis positive upward from the seabed surface.

According to the linear short-crested wave system (Hsu et al., 1979), we have the wave profile for partial reflection system as

$$
\eta=\frac{H_{\mathrm{i}}}{2}\left[\mathrm{e}^{\mathrm{i}(\omega t-m k x-n k y)}+K_{\mathrm{r}} \mathrm{e}^{\mathrm{i}(\omega t-m k x+n k y)}\right],
$$

where $H_{\mathrm{i}}$ is the incident wave height, $K_{\mathrm{r}}$ is the reflection coefficient, $\omega(=2 \pi / T, T$ is the wave period) is the wave frequency, and $k(=2 \pi / L, L$ is the wavelength) is the wave number of incident wave. As shown in Fig. $1, L_{x}$ and $L_{y}$ are the wavelengths in the direction of wave propagation (i.e. $x$-axis) and in the direction normal to the

(a)

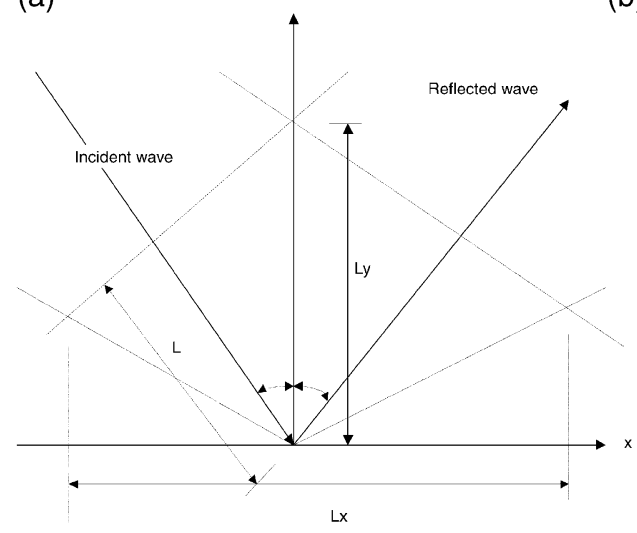

(b)

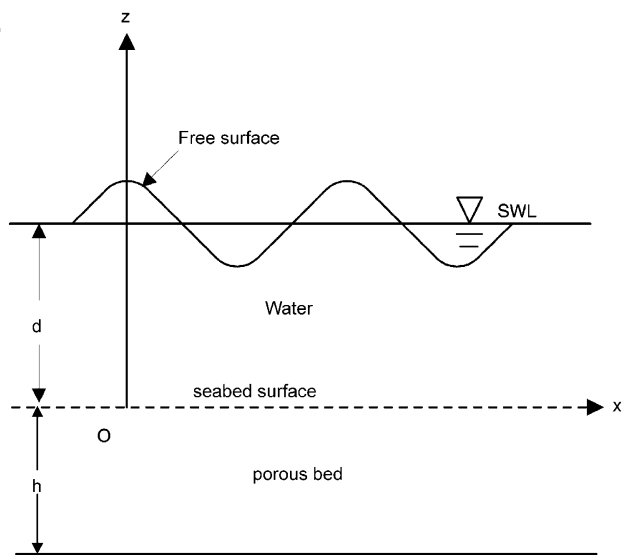

Fig. 1. Sketch of a short-crested wave system. 
wall (i.e. $y$-axis), respectively. The relationship between the wavelength of the incident wave $(L)$ and the wavelengths in horizontal and normal directions $\left(L_{x}\right.$ and $\left.L_{y}\right)$ are (Hsu et al., 1979)

$$
\frac{L}{L_{x}}=\sin \theta=m \quad \text { and } \quad \frac{L}{L_{y}}=\cos \theta=n .
$$

Since the water is considered as an incompressible fluid, and the wave field is an irrotational flow, the velocity potential $(\varphi)$ satisfies the conservation of mass, and lead to continuity equation, as

$$
\nabla^{2} \varphi=0 .
$$

The velocity potential $(\varphi)$ satisfies the above Laplace equation and the following free surface boundary conditions

$$
\begin{aligned}
& g \eta+\frac{\partial \varphi}{\partial t}=0, \\
& \frac{\partial \varphi}{\partial t}=\frac{\partial \eta}{\partial t},
\end{aligned}
$$

will have the general form as (Jeng, 2002)

$$
\begin{aligned}
\varphi= & \frac{\mathrm{i} g H_{\mathrm{i}}}{2 \omega}\left\{\cosh k(z-h)+\frac{\omega}{g k} \sinh k(z-h)\right\} \\
& \times\left\{\left(1+K_{\mathrm{r}}\right) \cos n k y-\left(1-K_{\mathrm{r}}\right) \sin n k y\right\} \mathrm{e}^{\mathrm{i}(\omega t-m k x)} .
\end{aligned}
$$

Here, we consider the seabed to be a coulomb-damped porous medium, the three-dimensional stress-strain relationships are

$$
\begin{aligned}
& \tau_{x x}^{\prime}=H \varepsilon-2 \mu\left(\varepsilon_{y y}+\varepsilon_{z z}\right)-C \xi, \\
& \tau_{y y}^{\prime}=H \varepsilon-2 \mu\left(\varepsilon_{x x}+\varepsilon_{z z}\right)-C \xi, \\
& \tau_{z z}^{\prime}=H \varepsilon-2 \mu\left(\varepsilon_{x x}+\varepsilon_{y y}\right)-C \xi, \\
& \tau_{y z}=\mu \varepsilon_{y z}, \\
& \tau_{x z}=\mu \varepsilon_{x z}, \\
& \tau_{x y}=\mu \varepsilon_{x y}, \\
& p=M \xi-C \varepsilon,
\end{aligned}
$$


where $\tau_{i j}$ are the stresses of the soil, $p$ is the pore pressure, and $\varepsilon$ is the volumetric strain, which is defined by

$$
\begin{aligned}
& \varepsilon=\varepsilon_{x x}+\varepsilon_{y y}+\varepsilon_{z z}, \\
& \varepsilon_{x x}=\frac{\partial u_{x}}{\partial x}, \\
& \varepsilon_{y y}=\frac{\partial u_{y}}{\partial y}, \\
& \varepsilon_{z z}=\frac{\partial u_{z}}{\partial z},
\end{aligned}
$$

and the shear strains are defined as

$$
\begin{aligned}
& \varepsilon_{y z}=\frac{\partial u_{y}}{\partial z}+\frac{\partial u_{z}}{\partial y}, \\
& \varepsilon_{x z}=\frac{\partial u_{x}}{\partial z}+\frac{\partial u_{z}}{\partial x}, \\
& \varepsilon_{x y}=\frac{\partial u_{y}}{\partial x}+\frac{\partial u_{x}}{\partial y},
\end{aligned}
$$

where $\vec{u} \equiv\left(u_{x}, u_{y}, u_{z}\right)$ is the solid displacement vector, and $\xi=-\nabla \cdot \vec{w}$ is the effective gradient of pore fluid, in which $\vec{w}=n_{\mathrm{e}}(\vec{U}-\vec{u}), n_{\mathrm{e}}$ is soil porosity, and $\vec{U} \equiv$ $\left(U_{x}, U_{y}, U_{z}\right)$ is the pore fluid displacements. In (6a)-(6g), $H, C, M$ and $\mu$ are the Biot's elastic constants (Biot, 1956), which take into account of the energy losses during each wave cyclic loading.

The equations of motion for solid particles and pore fluid give

$$
\begin{aligned}
& \mu \nabla^{2} \vec{u}+(H-\mu) \nabla \varepsilon-C \nabla \xi=\frac{\partial^{2}}{\partial t^{2}}\left(\rho \vec{u}+\rho_{\mathrm{f}} \vec{w}\right), \\
& \nabla(C \varepsilon+M \xi)=\frac{\partial^{2}}{\partial t^{2}}(\rho \vec{u}+m \vec{w})+\frac{\eta_{\mathrm{f}}}{k_{\mathrm{s}}} \frac{\partial \vec{w}}{\partial t},
\end{aligned}
$$

where the physical quantities are defined as (Lin, 2001):

$$
\begin{aligned}
& H=\frac{\left(K_{\mathrm{r}}-K_{\mathrm{s}}\right)^{2}}{D_{\mathrm{r}}-K_{\mathrm{s}}}+K_{\mathrm{s}}+\frac{4}{3} \mu, \quad C=\frac{K_{\mathrm{r}}\left(K_{\mathrm{r}}-K_{\mathrm{s}}\right)}{D_{\mathrm{r}}-K_{\mathrm{s}}}, \\
& M=\frac{K_{\mathrm{r}}^{2}}{D_{\mathrm{r}}-K_{\mathrm{s}}}, \quad D_{\mathrm{r}}=K_{\mathrm{r}}\left[1+n_{\mathrm{e}}\left(\frac{K_{\mathrm{r}}}{K_{\mathrm{f}}}-1\right)\right], \\
& \mu=G(1+\mathrm{i} \delta), \quad K_{\mathrm{s}}=K_{\mathrm{so}}(1+\mathrm{i} \delta), \\
& \rho=\left(1-n_{\mathrm{e}}\right) \rho_{\mathrm{r}}+n_{\mathrm{e}} \rho_{\mathrm{f}}, \quad m=(1+\alpha) \rho_{\mathrm{f}} / n_{\mathrm{e}},
\end{aligned}
$$

in which $\alpha$ is the added mass coefficient of the skeletal frame, $K_{\mathrm{r}}$ is the bulk modulus of the grain, $K_{\mathrm{f}}$ is the apparent bulk modulus of the pore fluid. The relationship 
between $K_{\mathrm{r}}$ and $K_{\mathrm{f}}$ is

$$
\frac{1}{K_{\mathrm{f}}}=\frac{1}{K_{\mathrm{fo}}}+\frac{1-S}{P_{\text {wo }}},
$$

where $S$ is the degree of saturation, $K_{\mathrm{fo}}=2 \times 10^{9} \mathrm{~N} / \mathrm{m}^{2}$, and $P_{\text {wo }}$ is the absolute static water pressures.

The above governing equations can be solved with the following boundary conditions:

- at the surface of the seabed $(z=0)$, we have

$$
\frac{\partial \varphi}{\partial z}=\frac{\partial u_{z}}{\partial t}+\frac{\partial w_{z}}{\partial t}, \quad p=-\rho_{\mathrm{f}} \frac{\partial \varphi}{\partial t}, \quad \tau_{z z}^{\prime}+p=0, \quad \tau_{x z}=\tau_{y z}=0
$$

- at the seabed bottom $(z=-d)$, we have

$$
\vec{u}=\vec{w}=0, \quad \frac{\partial p}{\partial z}=0
$$

\section{Analytical solutions}

To solve the soil displacements and pore fluid displacements in the governing equations (9a) and (9b) and boundary conditions (12) and (13), we let

$$
\begin{aligned}
& \vec{u}=\nabla \phi+\nabla \times \vec{\Phi}, \\
& \vec{w}=\nabla \psi+\nabla \times \vec{\Psi},
\end{aligned}
$$

from which the equation of motion can be re-written as

$$
\begin{aligned}
& \nabla^{2}(H \phi+C \psi)=\frac{\partial^{2}}{\partial t^{2}}\left(\rho \phi+\rho_{\mathrm{f}} \psi\right) \\
& \nabla^{2}(C \phi+M \psi)=\frac{\partial^{2}}{\partial t^{2}}\left(\rho_{\mathrm{f}} \phi+m \psi\right), \\
& \mu \nabla^{2} \vec{\Phi}=\frac{\partial^{2}}{\partial t^{2}}\left(\rho \vec{\Phi}+\rho_{\mathrm{f}} \vec{\Psi}\right) \\
& -\left(\frac{\eta_{\mathrm{f}}}{k_{\mathrm{s}}}\right) \frac{\partial \vec{\Psi}}{\partial t}=\frac{\partial^{2}}{\partial t^{2}}\left(\rho_{\mathrm{f}} \vec{\Phi}+m \vec{\Psi}\right) .
\end{aligned}
$$

Since the wave loading is periodical loading, we can set the form of the solution as

$$
\begin{aligned}
& \phi=\phi_{\mathrm{i}}(z) \mathrm{e}^{\mathrm{i}(\omega t-m k x-n k y)}+\phi_{\mathrm{r}}(z) \mathrm{e}^{\mathrm{i}(\omega t-m k x+n k y)}, \\
& \psi=\psi_{\mathrm{i}}(z) \mathrm{e}^{\mathrm{i}(\omega t-m k x-n k y)}+\psi_{\mathrm{r}}(z) \mathrm{e}^{\mathrm{i}(\omega t-m k x+n k y)}, \\
& \vec{\Phi}=\vec{\Phi}_{\mathrm{i}}(z) \mathrm{e}^{\mathrm{i}(\omega t-m k x-n k y)}+\vec{\Phi}_{\mathrm{r}}(z) \mathrm{e}^{\mathrm{i}(\omega t-m k x+n k y)}, \\
& \vec{\Psi}=\vec{\Psi}_{\mathrm{i}}(z) \mathrm{e}^{\mathrm{i}(\omega t-m k x-n k y)}+\vec{\Psi}_{\mathrm{r}}(z) \mathrm{e}^{\mathrm{i}(\omega t-m k x+n k y)} .
\end{aligned}
$$


Substituting the above relations into the governing equations, we have the incident wave components as

$$
\begin{aligned}
& \phi_{\mathrm{i}}=\left(a_{11} \mathrm{e}^{\lambda_{1} z}+a_{12} \mathrm{e}^{-\lambda_{1} z}+a_{21} \mathrm{e}^{\lambda_{2} z}+a_{22} \mathrm{e}^{-\lambda_{2} z}\right) \mathrm{e}^{\mathrm{i}(\omega t-n k y-m k x)}, \\
& \psi_{\mathrm{i}}=\left(b_{11} \mathrm{e}^{\lambda_{1} z}+b_{12} \mathrm{e}^{-\lambda_{1} z}+b_{21} \mathrm{e}^{\lambda_{2} z}+b_{22} \mathrm{e}^{-\lambda_{2} z}\right) \mathrm{e}^{\mathrm{i}(\omega t-n k y-m k x)}, \\
& \Phi_{\mathrm{i}}=\left(a_{31} \mathrm{e}^{\lambda_{3} z}+a_{32} \mathrm{e}^{-\lambda_{3} z}\right) \mathrm{e}^{\mathrm{i}(\omega t-n k y-m k x)}, \\
& \Psi_{\mathrm{i}}=\left(b_{31} \mathrm{e}^{\lambda_{3} z}+b_{32} \mathrm{e}^{-\lambda_{3} z}\right) \mathrm{e}^{\mathrm{i}(\omega t-n k y-m k x)},
\end{aligned}
$$

where

$$
\begin{aligned}
b_{1 i}= & c_{1} a_{1 i}, \quad b_{2 i}=c_{2} a_{2 i}, \quad b_{3 i}=c_{3} a_{3 i} \quad(i=1,2), \\
\lambda_{1}^{2}= & k^{2}-k_{\mathrm{f}}^{2}, \quad \lambda_{2}^{2}=k^{2}-k_{\mathrm{s}}^{2}, \quad \lambda_{3}^{2}=k^{2}-k_{\mathrm{T}}^{2}, \\
c_{1}= & -\frac{H-\rho V_{\mathrm{f}}}{C-\rho_{\mathrm{f}} V_{\mathrm{f}}}, \quad c_{2}=-\frac{H-\rho V_{\mathrm{s}}}{C-\rho_{\mathrm{f}} V_{\mathrm{s}}}, \quad c_{3}=-\frac{\rho_{\mathrm{f}}}{m_{1}}, \\
k_{\mathrm{f}}^{2}= & \left(\frac{\omega}{V_{\mathrm{f}}}\right)^{2}, \quad k_{\mathrm{s}}^{2}=\left(\frac{\omega}{V_{\mathrm{s}}}\right)^{2}, \quad k_{\mathrm{T}}^{2}=\frac{\omega^{2}\left(\rho-\rho_{\mathrm{f}}^{2} / m_{1}\right)}{\mu}, \\
V_{\mathrm{f}}^{2}= & 2\left(H M-C^{2}\right)\left[\left(\rho M+m_{1} H-2 \rho_{\mathrm{f}} C\right)\right. \\
& \left.+\sqrt{\left(m_{1} H-\rho M\right)^{2}+4 \rho_{\mathrm{f}}\left(\rho_{\mathrm{f}} M-m_{1} C\right) H+4 \rho\left(m_{1} C-\rho_{\mathrm{f}} M\right) C}\right]^{-1}, \\
m_{1}= & m-\mathrm{i} \frac{\eta_{\mathrm{f}}}{\omega k_{\mathrm{s}}} . \\
V_{\mathrm{s}}^{2}= & 2\left(H M-C^{2}\right)\left[\left(\rho M+m_{1} H-2 \rho_{\mathrm{f}} C\right)\right. \\
& -\sqrt{\left.\left(m_{1} H-\rho M\right)^{2}+4 \rho_{\mathrm{f}}\left(\rho_{\mathrm{f}} M-m_{1} C\right) H+4 \rho\left(m_{1} C-\rho_{\mathrm{f}} M\right) C\right]^{-1}},
\end{aligned}
$$


With the boundary conditions (12) and (13), we have the wave-induced soil response as

$$
\begin{aligned}
& p=\left\{\left(a_{11} \mathrm{e}^{\lambda_{1} z}+a_{12} \mathrm{e}^{-\lambda_{1} z}\right)\left(C+c_{1} M\right) k_{\mathrm{f}}^{2}+\left(a_{21} \mathrm{e}^{\lambda_{2} z}+a_{22} \mathrm{e}^{-\lambda_{2} z}\right)\left(C+c_{2} M\right) k_{\mathrm{s}}^{2}\right\} \\
& \times\left[\left(1+K_{\mathrm{r}}\right) \cos n k y-\mathrm{i}\left(1-K_{\mathrm{r}}\right) \sin n k y\right] \mathrm{e}^{\mathrm{i}(\omega t-m k x)}, \\
& \tau_{x x}=\left\{\left(a_{11} \mathrm{e}^{\lambda_{1} z}+a_{12} \mathrm{e}^{-\lambda_{1} z}\right)\left[-\left(H+c_{1} C\right) k_{\mathrm{f}}^{2}-2 \mu \lambda_{3}^{2}+2 \mu n^{2} k^{2}\right]\right. \\
& +\left(a_{21} \mathrm{e}^{\lambda_{2} z}+a_{22} \mathrm{e}^{-\lambda_{2} z}\right)\left[-\left(H+c_{2} C\right) k_{\mathrm{s}}^{2}-2 \mu \lambda_{3}^{2}+2 \mu n^{2} k^{2}\right] \\
& \left.-2 \mathrm{i} \mu m k \lambda_{3}\left(a_{31} \mathrm{e}^{\lambda_{2} z}-a_{32} \mathrm{e}^{-\lambda_{2} z}\right)\right\} \\
& \times\left[\left(1+K_{\mathrm{r}}\right) \cos n k y-\mathrm{i}\left(1-K_{\mathrm{r}}\right) \sin n k y\right] \mathrm{e}^{\mathrm{i}(\omega t-m k x)}, \\
& \tau_{y y}=\left\{\left(a_{11} \mathrm{e}^{\lambda_{1} z}+a_{12} \mathrm{e}^{-\lambda_{1} z}\right)\left[-\left(H+c_{1} C\right) k_{\mathrm{f}}^{2}-2 \mu \lambda_{3}^{2}+2 \mu m^{2} k^{2}\right]\right. \\
& +\left(a_{21} \mathrm{e}^{\lambda_{2} z}+a_{22} \mathrm{e}^{-\lambda_{2} z}\right)\left[-\left(H+c_{2} C\right) k_{\mathrm{s}}^{2}-2 \mu \lambda_{3}^{2}+2 \mu m^{2} k^{2}\right] \\
& \left.-2 \mathrm{i} \mu n k \lambda_{3}\left(a_{31} \mathrm{e}^{\lambda_{2} z}+a_{32} \mathrm{e}^{-\lambda_{2} z}\right)\right\} \\
& \times\left[\left(1+K_{\mathrm{r}}\right) \cos n k y-\mathrm{i}\left(1-K_{\mathrm{r}}\right) \sin n k y\right] \mathrm{e}^{\mathrm{i}(\omega t-m k x)}, \\
& \tau_{z z}=\left\{\left(a_{11} \mathrm{e}^{\lambda_{1} z}+a_{12} \mathrm{e}^{-\lambda_{1} z}\right)\left[-\left(H+c_{1} C\right) k_{\mathrm{f}}^{2}+2 \mu k^{2}\right]+\left(a_{21} \mathrm{e}^{\lambda_{2} z}+a_{22} \mathrm{e}^{-\lambda_{2} z}\right)\right. \\
& \left.\times\left[-\left(H+c_{2} C\right) k_{\mathrm{s}}^{2}+2 \mu k^{2}\right]-2 \mathrm{i} \mu m k \lambda_{3}\left(a_{31} \mathrm{e}^{\lambda_{2} z}+a_{32} \mathrm{e}^{-\lambda_{2} z}\right)\right\} \\
& \times\left[\left(1+K_{\mathrm{r}}\right) \cos n k y-\mathrm{i}\left(1-K_{\mathrm{r}}\right) \sin n k y\right] \mathrm{e}^{\mathrm{i}(\omega t-m k x)}, \\
& \tau_{x z}=\left\{2 \mathrm{i} \mu m k \lambda_{1}\left(-a_{11} \mathrm{e}^{\lambda_{1} z}+a_{12} \mathrm{e}^{-\lambda_{1} z}\right)\right]+2 \mathrm{i} \mu n k \lambda_{2}\left(-a_{21} \mathrm{e}^{\lambda_{2} z}+a_{22} \mathrm{e}^{-\lambda_{2} z}\right) \\
& \left.-\mu\left(m^{2} k^{2}+\lambda_{3}\right)\left(a_{31} \mathrm{e}^{\lambda_{2} z}+a_{32} \mathrm{e}^{-\lambda_{2} z}\right)\right\} \times\left[\left(1+K_{\mathrm{r}}\right) \cos n k y\right. \\
& \left.-\mathrm{i}\left(1-K_{\mathrm{r}}\right) \sin n k y\right] \mathrm{e}^{\mathrm{i}(\omega t-m k x)} \text {, } \\
& \tau_{y z}=\left\{2 \mu n k \lambda_{1}\left(a_{11} \mathrm{e}^{\lambda_{1} z}-a_{12} \mathrm{e}^{-\lambda_{1} z}\right)\right]+2 \mu n k \lambda_{2}\left(a_{21} \mathrm{e}^{\lambda_{2} z}-a_{22} \mathrm{e}^{-\lambda_{2} z}\right) \\
& \left.-\mathrm{i} \mu m n k^{2}\left(a_{31} \mathrm{e}^{\lambda_{2} z}-a_{32} \mathrm{e}^{-\lambda_{2} z}\right)\right\} \times\left[-\left(1+K_{\mathrm{r}}\right) \sin n k y\right. \\
& \left.+\mathrm{i}\left(1-K_{\mathrm{r}}\right) \cos n k y\right] \mathrm{e}^{\mathrm{i}(\omega t-m k x)}, \\
& \left.\tau_{x y}=\left\{-2 \mathrm{i} \mu m n k^{2}\left(a_{11} \mathrm{e}^{\lambda_{1} z}+a_{12} \mathrm{e}^{-\lambda_{1} z}\right)\right]-2 \mathrm{i} \mu m n k^{2}\left(a_{21} \mathrm{e}^{\lambda_{2} z}+a_{22} \mathrm{e}^{-\lambda_{2} z}\right)\right\} \\
& \times\left[-\left(1+K_{\mathrm{r}}\right) \sin n k y+\mathrm{i}\left(1-K_{\mathrm{r}}\right) \cos n k y\right] \mathrm{e}^{\mathrm{i}(\omega t-m k x)},
\end{aligned}
$$

where $a_{i j}$ satisfy

$$
\begin{gathered}
{[A][X]=[B],} \\
{[A]=\left[\begin{array}{llllll}
\gamma_{1} & \gamma_{1} & \gamma_{2} & \gamma_{2} & -A_{1} & A_{1} \\
-A_{2} & A_{2} & -A_{3} & A_{3} & -A_{4} & -A_{4} \\
A_{5} & A_{5} & A_{6} & A_{6} & 0 & 0 \\
B_{1} \mathrm{e}^{-\lambda_{1} d} & B_{1} \mathrm{e}^{\lambda_{1} d} & B_{1} \mathrm{e}^{-\lambda_{2} d} & B_{1} \mathrm{e}^{\lambda_{2} d} & -\lambda_{3} \mathrm{e}^{-\lambda_{3} d} & \lambda_{3} \mathrm{e}^{\lambda_{3} d} \\
\lambda_{1} \mathrm{e}^{-\lambda_{1} d} & -\lambda_{1} \mathrm{e}^{-\lambda_{1} d} & \lambda_{2} \mathrm{e}^{-\lambda_{2} d} & -\lambda_{2} \mathrm{e}^{\lambda_{2} d} & -\mathrm{i} k \mathrm{e}^{-\lambda_{3} d} & -\mathrm{i} k \mathrm{e}^{\lambda_{3} d} \\
c_{1} \lambda_{1} \mathrm{e}^{-\lambda_{1} d} & -c_{1} \lambda_{1} \mathrm{e}^{\lambda_{1} d} & c_{2} \lambda_{2} \mathrm{e}^{-\lambda_{2} d} & -c_{2} \lambda_{2} \mathrm{e}^{\lambda_{2} d} & -\mathrm{i} k c_{3} \mathrm{e}^{-\lambda_{3} d} & -\mathrm{i} k c_{3} \mathrm{e}^{\lambda_{3} d}
\end{array}\right],}
\end{gathered}
$$




$$
[X]=\left[\begin{array}{l}
a_{11} \\
a_{12} \\
a_{21} \\
a_{22} \\
a_{31} \\
a_{32}
\end{array}\right] \quad \text { and } \quad[B]=\left[\begin{array}{l}
0 \\
0 \\
-\rho_{\mathrm{f}} \\
0 \\
0 \\
0 \\
0
\end{array}\right],
$$

where

$$
\begin{aligned}
& \gamma_{1}=k_{\mathrm{s}}^{2}\left[(H-C)+(C-M) c_{1}\right]+2 \mu k^{2}, \\
& \gamma_{2}=k_{\mathrm{f}}^{2}\left[(H-C)+(C-M) c_{2}\right]+2 \mu k^{2}, \\
& A_{1}=2 \mathrm{i} \mu m k \lambda_{3}, \quad A_{2}=2 \mathrm{i} \mu m k \lambda_{1}, \quad A_{3}=2 \mathrm{i} \mu m k \lambda_{2}, \\
& A_{4}=\mu\left(m^{2} k^{2}-\lambda_{3}^{2}\right), \quad A_{5}=\left(M c_{1}+C\right) k_{\mathrm{f}}^{2}, \quad A_{6}=\left(M c_{2}+C\right) k_{\mathrm{s}}^{2}, \\
& B_{1}=-\mathrm{i} m k
\end{aligned}
$$

\section{Results and discussions}

The aims of this paper are to (1) extend the previous two-dimensional solution to three-dimensional case with Columb-damping friction, and (2) examine the waveinduced liquefaction and shear failure potential. Based on the analytical solutions presented above, some numerical examples will be illustrated in the following sections.

\subsection{Wave-induced stresses in a porous seabed}

In this section, we consider $\theta=30^{\circ}$ as an example. The vertical distributions of the wave-induced pore pressure, effective normal stresses and shear stresses versus soil depth at different location, $y / L_{y}=0,1 / 12$ and $1 / 6$ are plotted in Figs. 2-8. In the figures, $p_{\mathrm{b}}\left(=\gamma_{\mathrm{w}} H / 2 \cosh k h\right)$ is the amplitude of dynamic wave pressure at the seabed surface. For comparison, the results of progressive wave $\left(\theta=90^{\circ}\right.$, in dashed lines) and standing wave $\left(\theta=0^{\circ}\right.$, in dashed-dotted lines) at $y / L_{y}=1 / 12$ are also plotted in the figures.

As shown in Fig. 2, the influence of distance from the breakwater $\left(y / L_{y}\right)$ on the wave-induced pore pressure is not obvious. Basically, the pore pressure $\left(p / p_{\mathrm{b}}\right)$ decreases as $y / L_{y}$ increases. However, the pore pressure does not changed as soil depth $(z / L)$ and distance from the breakwater $\left(y / L_{y}\right)$ change, when $z / L>0.3$. On the other hand, the influence of $y / L_{y}$ on the wave-induced vertical effective stress $\left(\tau_{z z}^{\prime} / p_{\mathrm{b}}\right)$ is more significant. The vertical effective stress $\tau_{z z}^{\prime} / p_{\mathrm{b}}$ increases as $y / L_{y}$ decreases (Fig. 3).

Compared with the pore pressure and vertical effective normal stress, the distance from the breakwater $\left(y / L_{y}\right)$ significantly affect the horizontal effective normal stresses $\left(\tau_{x x}^{\prime} / p_{\mathrm{b}}\right.$ and $\left.\tau_{y y}^{\prime} / p_{\mathrm{b}}\right)$, as illustrated in Figs. 4 and 5. In general, $\tau_{x x}^{\prime} / p_{\mathrm{b}}$ and $\tau_{y y}^{\prime} / p_{\mathrm{b}}$ decreases as $y / L_{y}$ increases. As shown in Fig. 4 , the short-crested wave-induced 


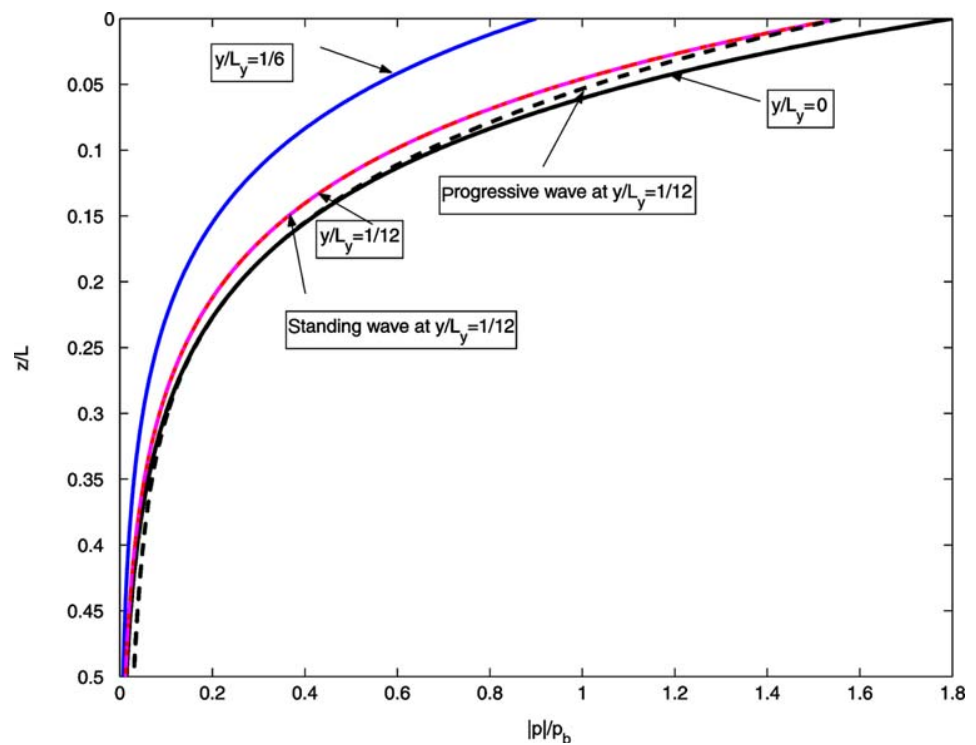

Fig. 2. Distribution of the wave-induced pore pressure $\left(|p| / p_{\mathrm{b}}\right)$ versus soil depth $(z / L)$ at different location $y / L_{y}\left(\theta=30^{\circ}\right.$ and $\left.K_{\mathrm{r}}=0.8\right)$.

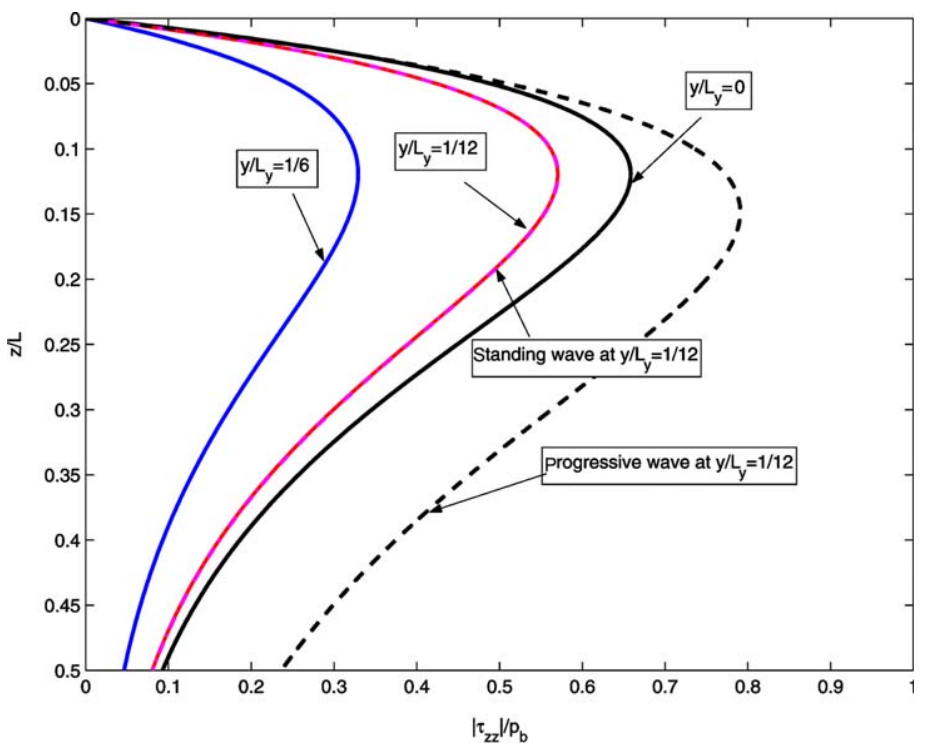

Fig. 3. Distribution of the wave-induced normal stress $\left(\left|\tau_{z z}^{\prime}\right| / p_{\mathrm{b}}\right)$ versus soil depth $(z / L)$ at different location $y / L_{y}\left(\theta=30^{\circ}\right.$ and $\left.K_{\mathrm{r}}=0.8\right)$. 


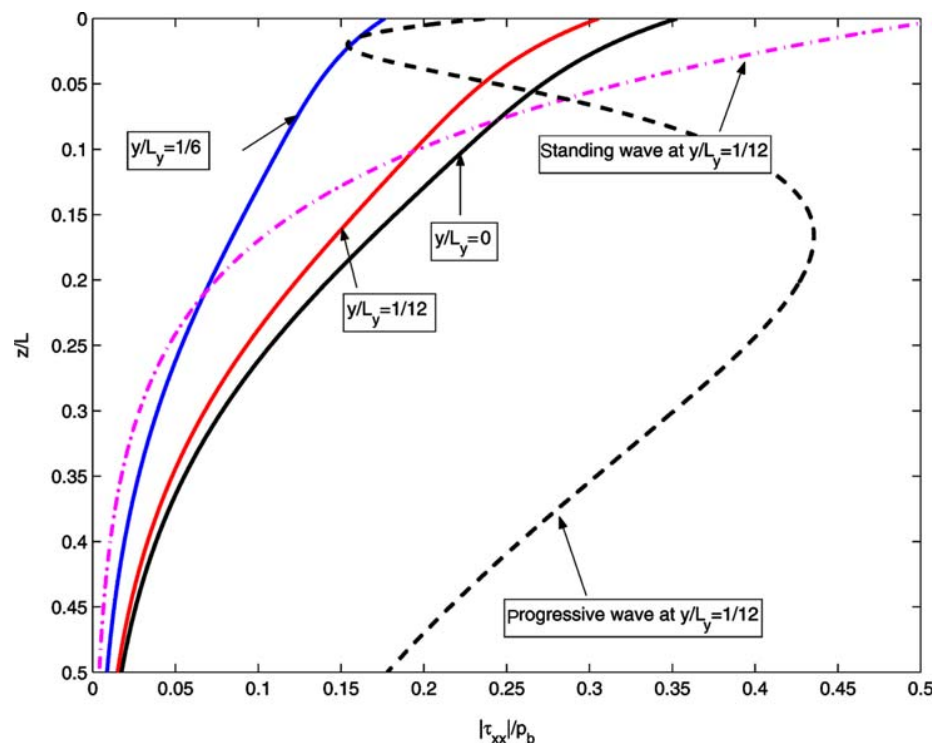

Fig. 4. Distribution of the wave-induced normal stress $\left(\left|\tau_{x x}^{\prime}\right| / p_{\mathrm{b}}\right)$ versus soil depth $(z / L)$ at different location $y / L_{y}\left(\theta=30^{\circ}\right.$ and $\left.K_{\mathrm{r}}=0.8\right)$.

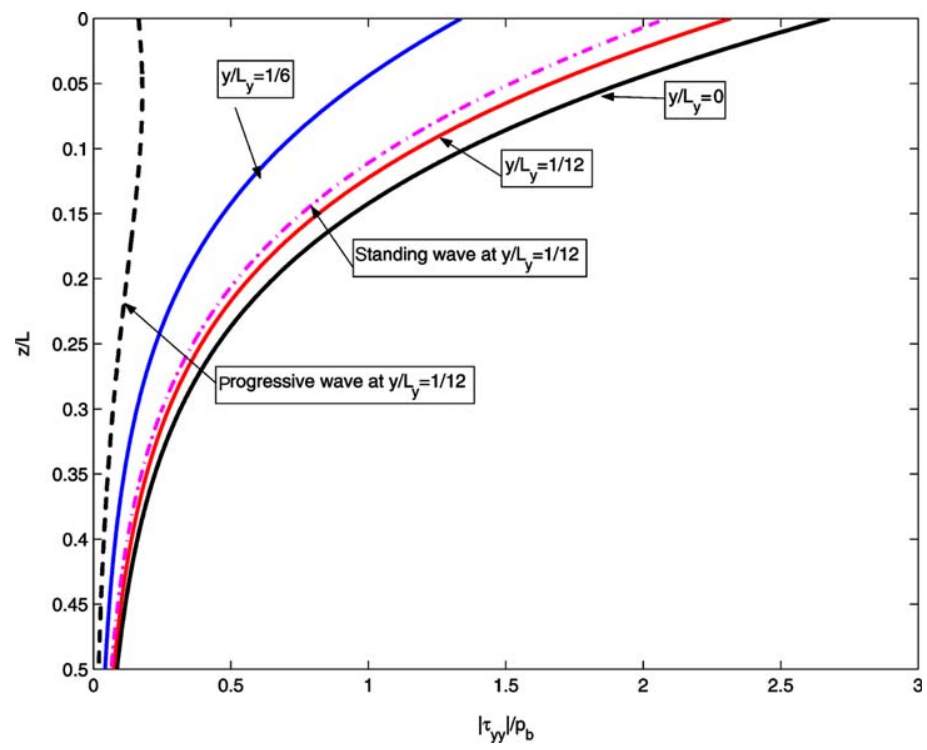

Fig. 5. Distribution of the wave-induced normal stress $\left(\left|\tau_{y y}^{\prime}\right| / p_{\mathrm{b}}\right)$ versus soil depth $(z / L)$ at different location $y / L_{y}\left(\theta=30^{\circ}\right.$ and $\left.K_{\mathrm{r}}=0.8\right)$. 


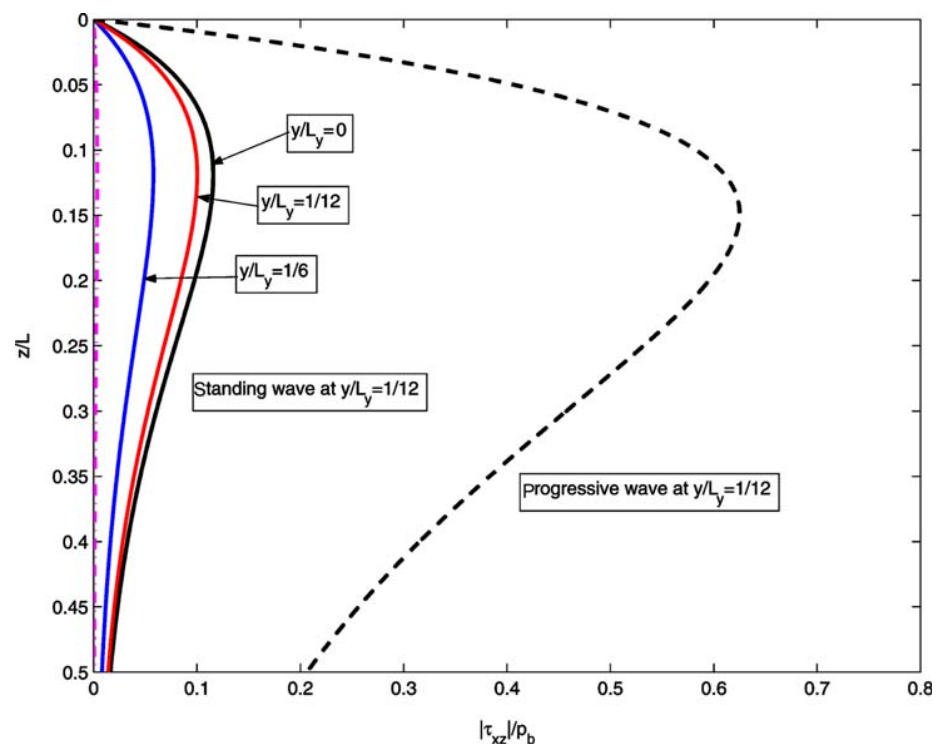

Fig. 6. Distribution of the wave-induced shear stress $\left(\left|\tau_{x z}^{\prime}\right| / p_{\mathrm{b}}\right)$ versus soil depth $(z / L)$ at different location $y / L_{y}\left(\theta=30^{\circ}\right.$ and $\left.K_{\mathrm{r}}=0.8\right)$.

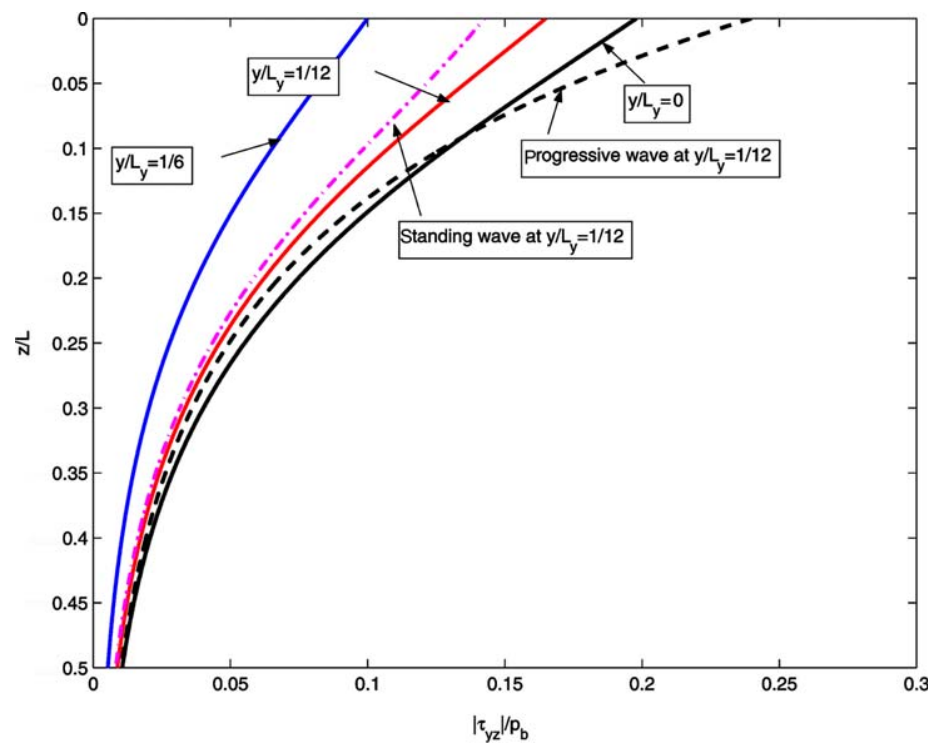

Fig. 7. Distribution of the wave-induced shear stress $\left(\left|\tau_{y z}^{\prime}\right| / p_{\mathrm{b}}\right)$ versus soil depth $(z / L)$ at different location $y / L_{y}\left(\theta=30^{\circ}\right.$ and $\left.K_{\mathrm{r}}=0.8\right)$. 


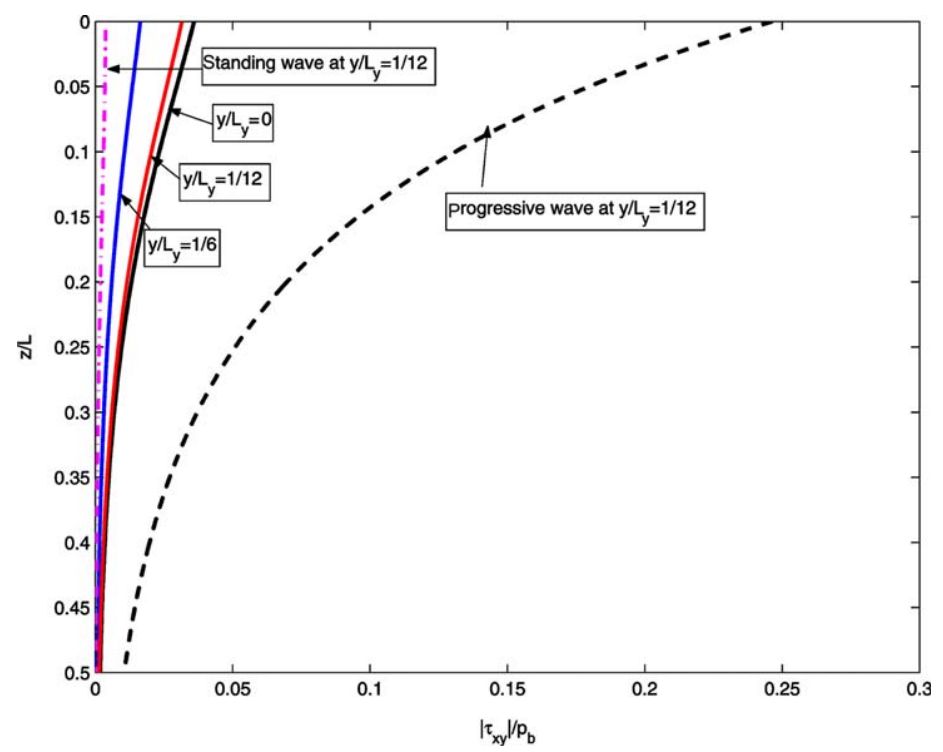

Fig. 8. Distribution of the wave-induced shear stress $\left(\left|\tau_{x y}^{\prime}\right| / p_{\mathrm{b}}\right)$ versus soil depth $(z / L)$ at different location $y / L_{y}\left(\theta=30^{\circ}\right.$ and $\left.K_{\mathrm{r}}=0.8\right)$.

$\tau_{x x}^{\prime} / p_{\mathrm{b}}$ is smaller than that induced by progressive and standing waves, when $z / L<0.05$. However, the results are revise, when $z / L>0.15$. On the other hand, the horizontal effective normal stress $\tau_{y y}^{\prime} / p_{\mathrm{b}}$ induced by short-crested waves is one order higher than that of standing waves (Fig. 5). Thus, it is necessary to pay extra attention to the short-crested waves-induced horizontal effective normal stresses.

Similar trend is observed for the wave-induced shear stresses. As shown in Figs. 6-8, the results of short-crested waves are not always located between progressive and standing waves, especially near the surface of the seabed. Thus, to investigate the influences of these physical quantities on the seabed instability, we cannot just rely on the magnitudes of two physical parameters, but considering the combined effects of all parameters.

\subsection{Wave-induced seabed instability}

In this study, we focus on the wave-induced liquefaction and shear failure potential. The definition of two failure modes are summarised here.

\subsubsection{Shear failure}

It has been well known that the shear stress at particular point of the seabed is greater than the shear strength of the soil will lead to shear failure. This kind of 
instability based on the spatial distribution of the wave-induced shear failure and the shear strength of the soil. The Mohr-Coulomb criterion has been commonly used to estimate the shear failure. Based on Mohr-Columb criterion, we have

$$
\bar{\tau}_{\mathrm{f}}=\bar{\sigma}_{\mathrm{f}} \tan \theta_{\mathrm{f}},
$$

where $\bar{\tau}_{\mathrm{f}}$ and $\bar{\sigma}_{\mathrm{f}}$ are the shear stress and normal stress at the failure plane. The internal friction angle $\theta_{\mathrm{f}}$ depends on the soil types. For example, $\theta_{\mathrm{f}}=35^{\circ}$ for medium sand, and $\theta_{\mathrm{f}}=30^{\circ}$ for fine sand. The stress state at any instant and location can be expressed in term of the stress angle $\theta$ as

$$
\sin \theta=\frac{\sigma_{1}-\sigma_{3}}{\sigma_{1}+\sigma_{3}}=\frac{\sqrt{3} t \cos \alpha}{t \sin \alpha-\sqrt{2} s} .
$$

The seabed will become unstable when $\theta(x, y, z ; t) \geq \theta_{\mathrm{f}}$.

In (31), the three-dimensional principal stresses can be determined by (Griffith, 1986)

$$
\begin{aligned}
& \sigma_{1}=\frac{s}{\sqrt{3}}+\sqrt{\frac{2}{3}} t \sin \left(\alpha-\frac{2 \pi}{3}\right), \quad \sigma_{2}=\frac{s}{\sqrt{3}}+\sqrt{\frac{2}{3}} t \sin \alpha, \\
& \sigma_{3}=\frac{s}{\sqrt{3}}+\sqrt{\frac{2}{3}} t \sin \left(\alpha+\frac{2 \pi}{3}\right), \quad s=\frac{1}{\sqrt{3}}\left(\bar{\tau}_{x x}+\bar{\tau}_{y y}+\tau_{z z}\right), \\
& t=\sqrt{\frac{\left(\bar{\tau}_{x x}-\bar{\tau}_{y y}\right)^{2}+\left(\bar{\tau}_{x x}-\bar{\tau}_{z z}\right)^{2}+\left(\bar{\tau}_{z z}-\bar{\tau}_{y y}\right)^{2}+6\left(\bar{\tau}_{x x}^{2}+\bar{\tau}_{y y}^{2}+\bar{\tau}_{z z}^{2}\right)}{3},} \\
& \alpha=\frac{1}{3} \sin ^{-}\left(-\frac{3 \sqrt{6} J_{3}}{t^{3}}\right), \\
& J_{3}=S_{x} S_{y} S_{z}-S_{x} \bar{\tau}_{y z}^{2}-S_{y} \bar{\tau}_{x x}^{2}-S_{z} \bar{\tau}_{x y}^{2}+2 \bar{\tau}_{x y} \bar{\tau}_{x z} \bar{\tau}_{y z}, \\
& S_{x}=\frac{2 \bar{\tau}_{x x}-\bar{\tau}_{y y}-\bar{\tau}_{z z}}{3}, \quad S_{y}=\frac{2 \bar{\tau}_{y y}-\bar{\tau}_{x x}-\bar{\tau}_{z z}}{3}, \\
& S_{z}=\frac{2 \bar{\tau}_{z z}-\bar{\tau}_{y y}-\bar{\tau}_{x x}}{3}, \\
& \bar{\tau}_{x x}=\tau_{x 0}-\tau_{x x}^{\prime}=-\left(\gamma_{\mathrm{s}}-\gamma_{\mathrm{w}}\right) K_{0} z-\tau_{x x}-p, \\
& \bar{\tau}_{y y}=\tau_{y 0}-\tau_{y y}^{\prime}=-\left(\gamma_{\mathrm{s}}-\gamma_{\mathrm{w}}\right) K_{0} z-\tau_{y y}-p, \\
& \bar{\tau}_{z z}=\tau_{z 0}-\tau_{z z}^{\prime y}=-\left(\gamma_{s}-\gamma_{\mathrm{w}}\right) z-\tau_{z z}-p, \\
& \bar{\tau}_{x z}=-\tau_{x z}, \quad \bar{\tau}_{y z}=-\tau_{y z}, \quad \bar{\tau}_{x y}=-\tau_{x y} .
\end{aligned}
$$

where $\gamma_{\mathrm{s}}$ and $\gamma_{\mathrm{w}}$ are the unit weight of soil and pore water, respectively, and $K_{0}$ is the lateral coefficient of earth pressure, which is defined by

$$
K_{0}=\frac{v}{1-v},
$$

in which $v$ is the Poisson's ratio.

\subsubsection{Liquefaction}

The failure mode of liquefaction is different from that of shear failure. The waveinduced dynamic stresses and strains cause the soil particles movement, and results in the volumetric change. This leads to the effective stress of solid transfer to pore 
pressure, and leads to the pore pressure increases and effective stress decreases. Under the limiting condition, the excess pore pressure will cause the effective stress to be zero, and then the shear strength of solid particle will no longer exist, which will cause liquefaction. Here, what we discuss is the instant liquefaction, or socalled initial liquefaction, which is the response of solid particles and pore water due to the wave loading. In principle, the instant liquefaction will not involve with the deformation after liquefaction, which is the fundamental of soil behavior, and will cause the instability of the offshore structure. Thus, the evaluation of the instant liquefaction of the soil is important for coastal engineers. Here, we use the three-dimensional liquefied criterion to analyses the initial liquefaction (Jeng, 1997):

$$
-\frac{1}{3}\left(\gamma_{\mathrm{s}}-\gamma_{\mathrm{w}}\right)\left(1+2 K_{0}\right) z+\left(p_{\mathrm{b}}-p\right) \leq 0
$$

where $\left(p_{\mathrm{b}}-p\right)$ is the excess pore pressure, $p_{\mathrm{b}}$ is the water pressure at the surface of the seabed, which varies as the wave propagation, and $p$ is the pore water pressure in the seabed.

In the following examples, we consider the wave-induced seabed instability in two different seabeds - coarse sand and fine sand. In the numerical examples, the reflection coefficient $K_{\mathrm{r}}$ is taken as 0.8 .

Figs. 9-12 illustrate the maximum liquefaction depth and shear failure depth versus wave period $(T)$ and water depth $(h / L)$. The figures clearly indicate that the liquefaction depth increases as wave period increases. In a coarse sand, the liquefaction does not vary with the incident angle, which agrees with the previous work (Jeng, 1997). However, in a fine sand, the liquefaction depth varies with the incident wave angles. The liquefaction depth occurs at $\theta=90^{\circ}$ is less than that at $\theta=60^{\circ}$, which emphasizes that the short-crested wave with large incident wave angle will cause deeper liquefied region than the progressive waves. However, the trends of shear failure for coarse and fine sand are different. For example, the maximum shear failure depth increases as the incident wave angle increase in coarse sand. However, the maximum shear failure occurs at $\theta=60^{\circ}$ in fine sand. It is also observed in Fig. 11 that the shear failure depth increases as the wave period increases, when $T>2$ s. This result overall agrees with the experimental results of Xie (1983) and Jan and Lin (1998).

It is also observed that the liquefaction decreases as the relative water depth $(h / L)$ increases in coarse sand. However, there is a peak value of relative water depth in fine sand. Figs. 10 and 11 indicate that the liquefaction depth in coarse sand is larger than that in fine sand, when incident wave angle $\theta \leq 45^{\circ}$. On the other hand, the liquefaction depth in fine sand is greater than that in coarse sand, when $\theta=60^{\circ}, T>6.6 \mathrm{~s}$ or $h / L>0.18$. Under such conditions, engineers must pay more attentions. Fig. 12 also indicates that the maximum shear failure depth decreases as the water depth increases, while the maximum shear failure depth occurs at $h / L=0.1$ in fine sand. 


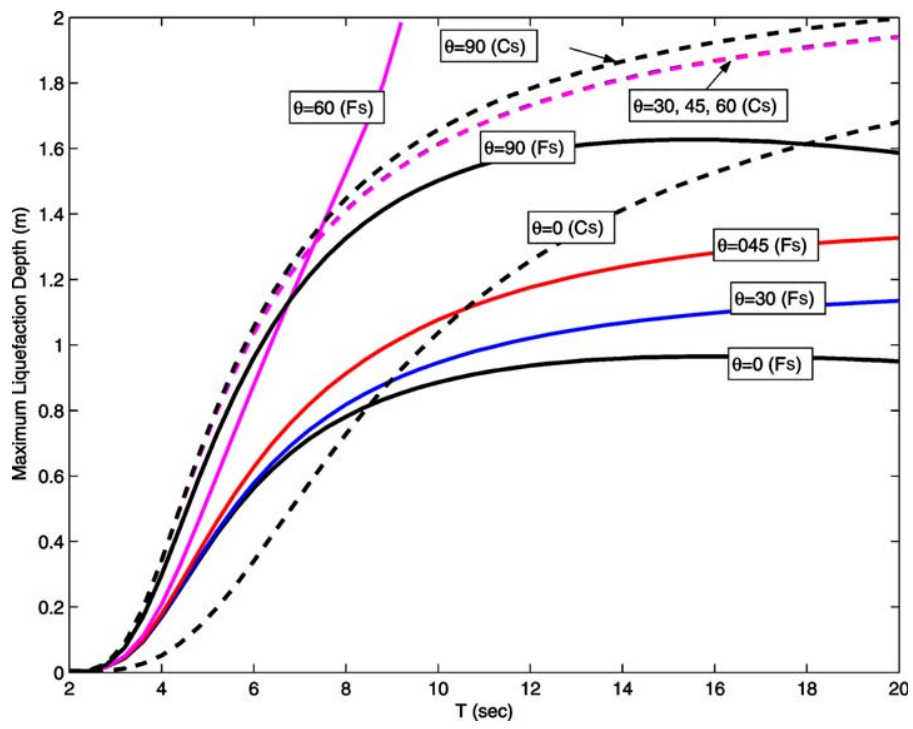

Fig. 9. Wave-induced maximum liquefaction depth versus wave period $(T)$ for various incident angles $(\theta)$. Solid lines, fine sand; dashed lines, coarse sand $\left(K_{\mathrm{r}}=0.8\right)$.

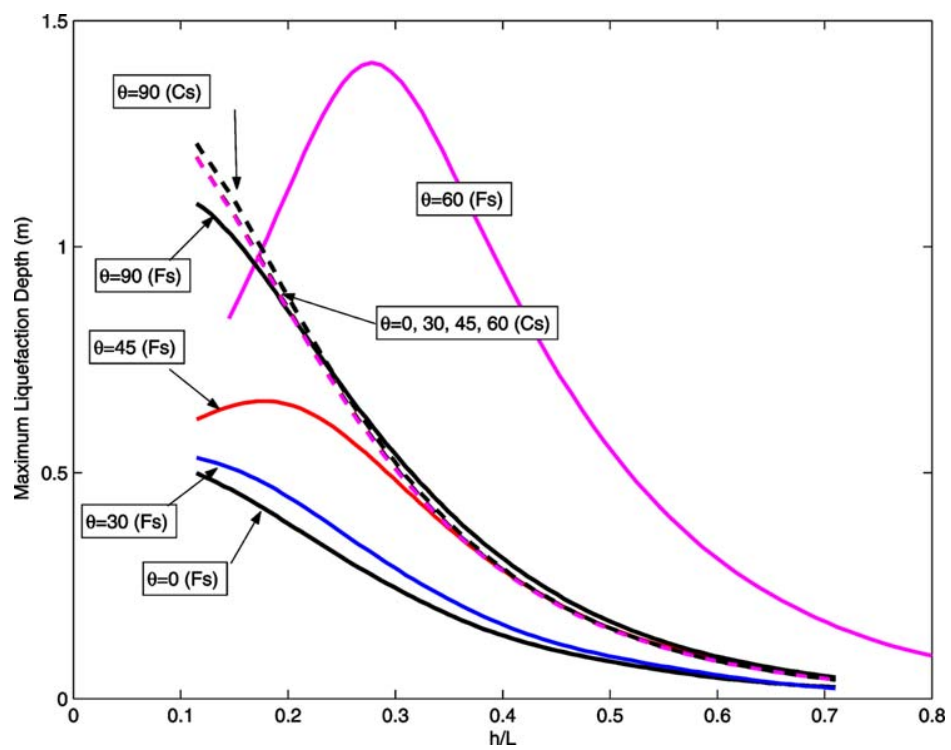

Fig. 10. Wave-induced maximum liquefaction depth versus relative water depth $(h / L)$ for various incident angles $(\theta)$. Solid lines, fine sand; dashed lines, coarse sand $\left(K_{\mathrm{r}}=0.8\right)$. 


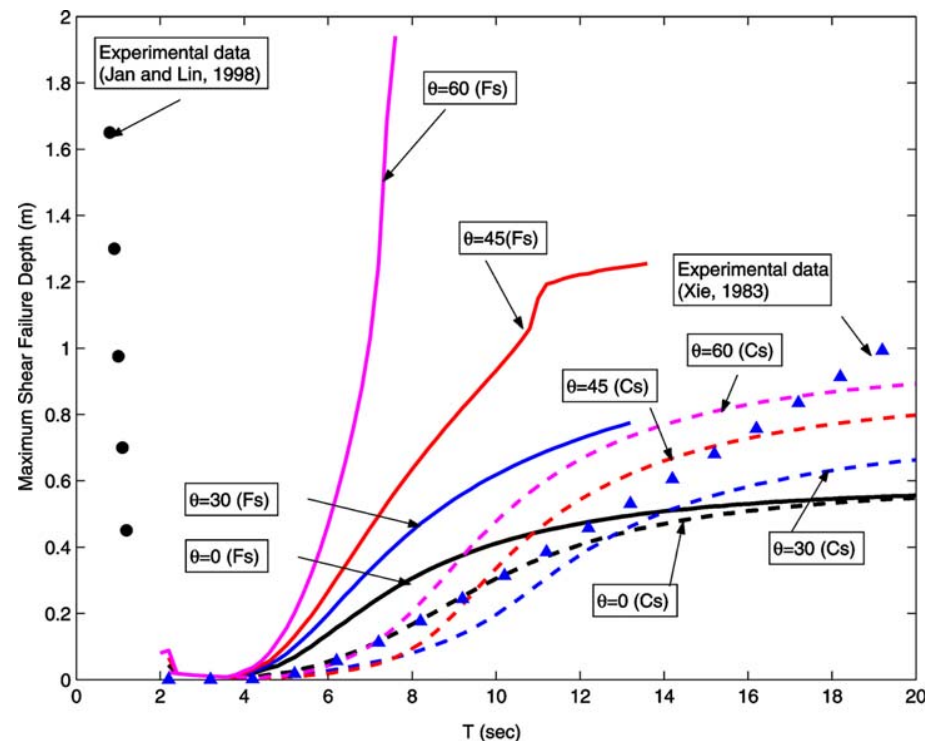

Fig. 11. Wave-induced maximum shear failure depth versus wave period $(T)$ for various incident angles $(\theta)$. Solid lines, fine sand; dashed lines, coarse sand $\left(K_{\mathrm{r}}=0.8\right)$.

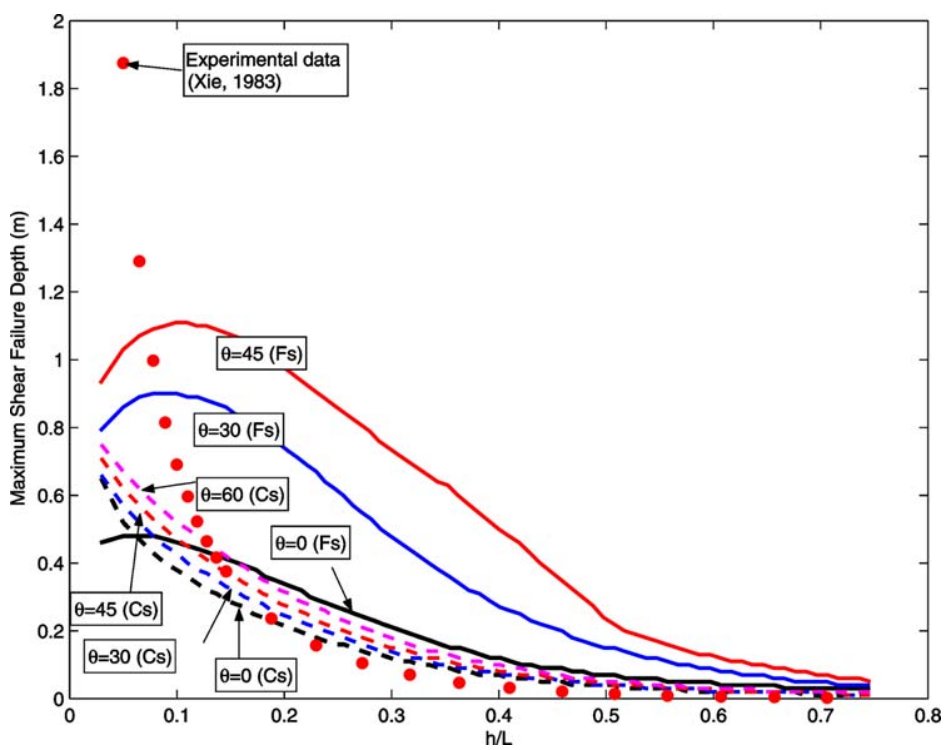

Fig. 12. Wave-induced maximum shear failure depth versus relative water depth $(h / L)$ for various incident angles $(\theta)$. Solid lines, fine sand; dashed lines, coarse sand $\left(K_{\mathrm{r}}=0.8\right)$. 


\subsection{Effects of reflection coefficients}

One of new contribution of this paper is the consideration of reflection coefficient, $K_{\mathrm{r}}$. Fig. 13 illustrates the influences of reflection coefficients on the waveinduced liquefaction and shear failure depth in fine sand. The figure clearly indicates that the liquefaction depth and shear failure depth increases as $K_{\mathrm{r}}$ increases. It is also observed that the incident wave angles does not significantly affect the liquefaction depth, while it significant affects the shear failure depth (Fig. 13).

\subsection{Effects of self-weight of a breakwater}

In realistic engineering problem, the self-weight of the breakwater must be taken into account. Considering a rectangular breakwater, the added stresses due to the self-weight of the breakwater, $\sigma_{x x}^{\prime}, \sigma_{y y}^{\prime}$ and $\sigma_{z z}^{\prime}$, are plotted in Figs. 14-16. In the figures, $s$ is the width of breakwater, which is taken as $20 \mathrm{~m}$ in the numerical examples, and $p_{\mathrm{b}}$ is the pressure at the bottom of the breakwater. As shown in Figs. 14 16 , the added stresses reduce to $10 \%$ of $p_{\mathrm{b}}$, when $y / s>1.8$. Figs. 17 and 18 further demonstrate that the added stresses is two order less than $p_{\mathrm{b}}$.

Figs. 19-22 further compare the wave-induced stresses without and with consideration of the self-weight of the breakwater. In the figures, the solid lines denotes the results with consideration of added stress, while the dashed lines represent the case without added stresses. Figs. 19-22 clearly indicate that the added stresses can be ignored when $y / L_{y}>1 / 4$. This conclusion can also be observed in

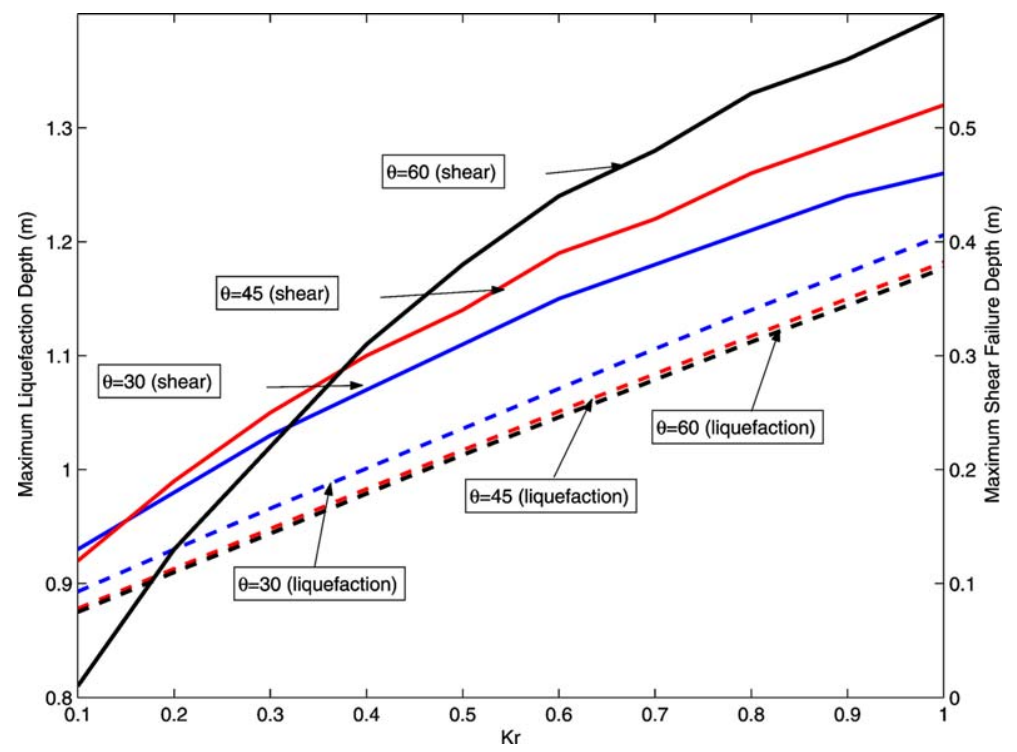

Fig. 13. Wave-induced maximum liquefaction and shear failure depth versus partial reflection coefficient $\left(K_{\mathrm{r}}\right)$ for various incident angles $(\theta)$ in fine sand. 


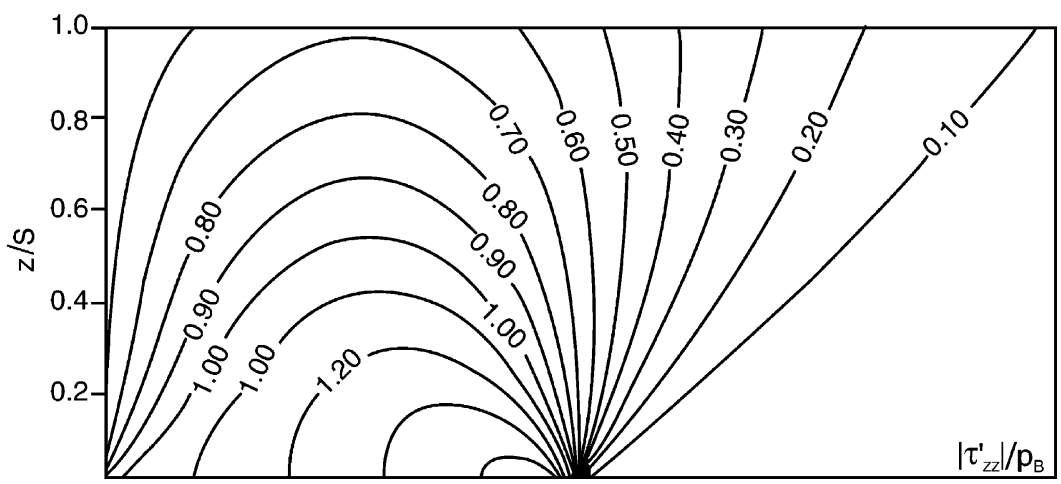

Fig. 14. Contour distribution of added stresses $\left(\tau_{z z} / p_{\mathrm{b}}\right)$ due to self-weight of the breakwater $(s=20 \mathrm{~m})$.

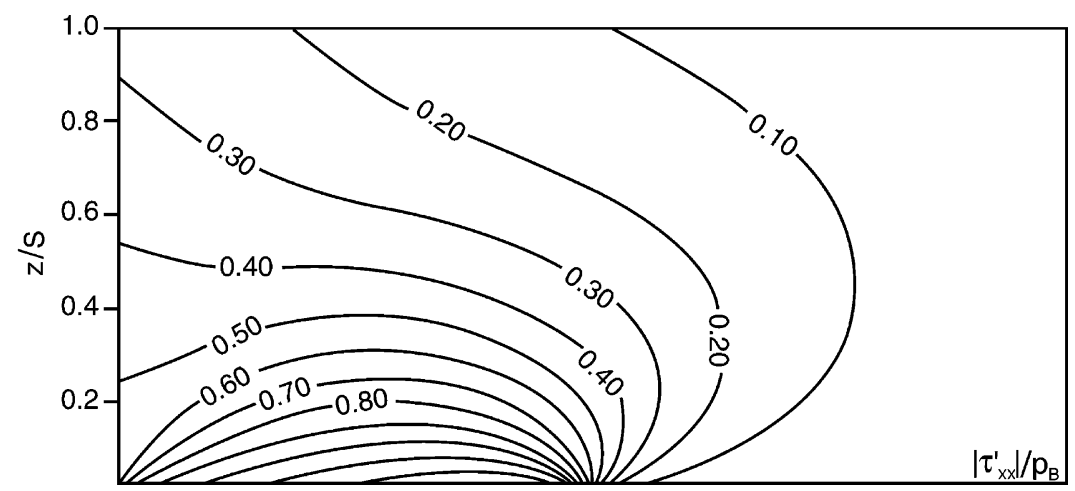

Fig. 15. Contour distribution of added stresses $\left(\tau_{x x} / p_{\mathrm{b}}\right)$ due to self-weight of the breakwater $(s=20 \mathrm{~m})$.

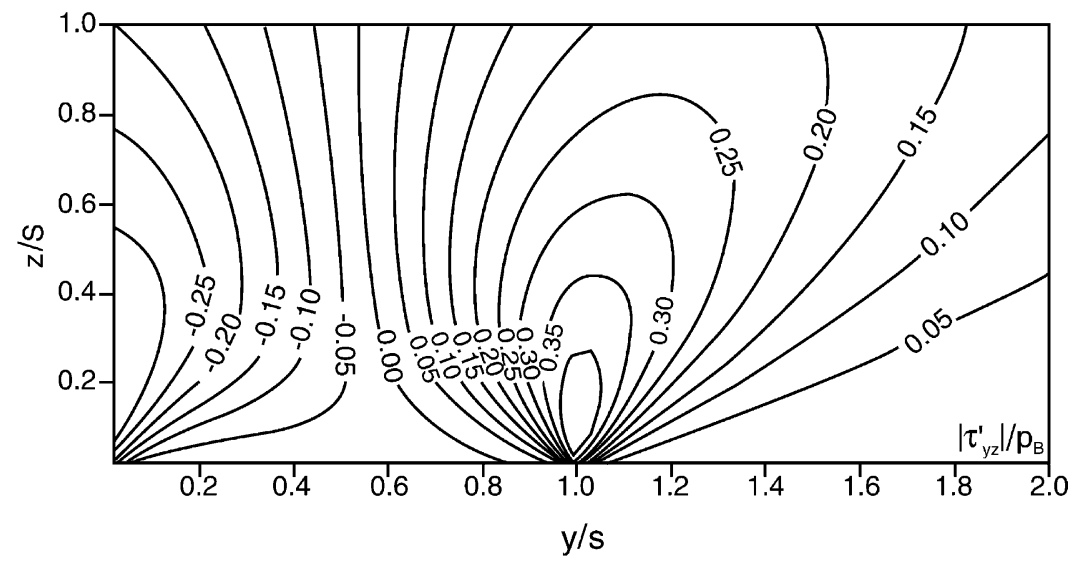

Fig. 16. Contour distribution of added stresses $\left(\tau_{y z} / p_{\mathrm{b}}\right)$ due to self-weight of the breakwater $(s=20 \mathrm{~m})$. 


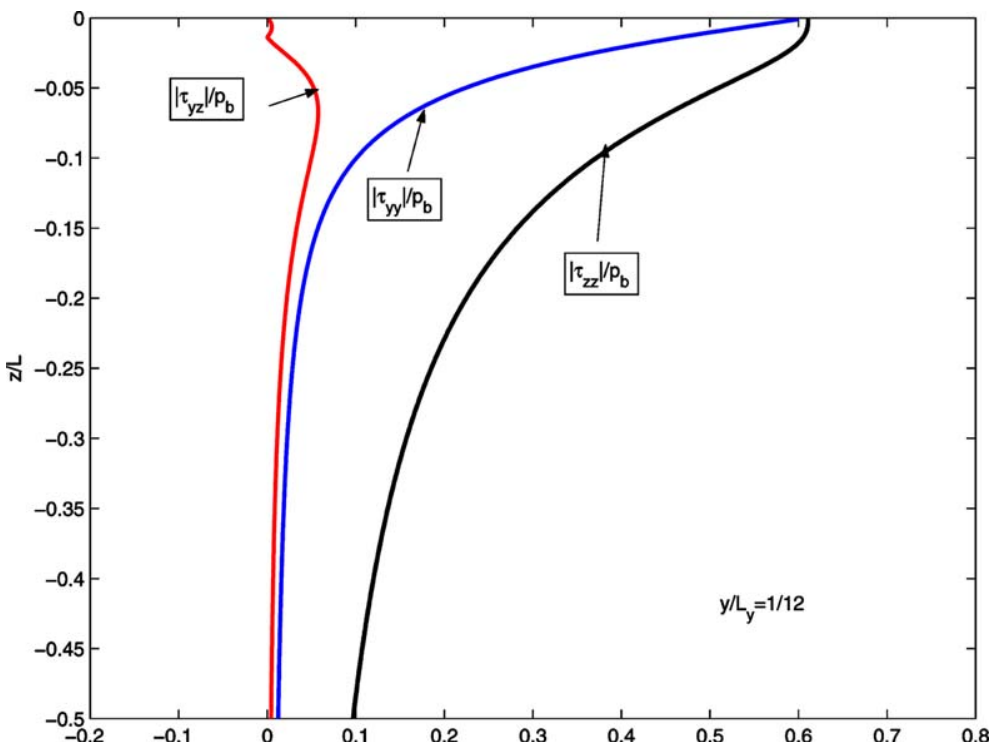

Fig. 17. Vertical distribution of added stresses, $\left|\tau_{y z}\right| / p_{\mathrm{b}}, \tau_{y y} / p_{\mathrm{b}}$ and $\left|\tau_{z z}\right| / p_{\mathrm{b}}$, due to self-weight of a breakwater at $y / L_{y}=1 / 12(s=20 \mathrm{~m})$.

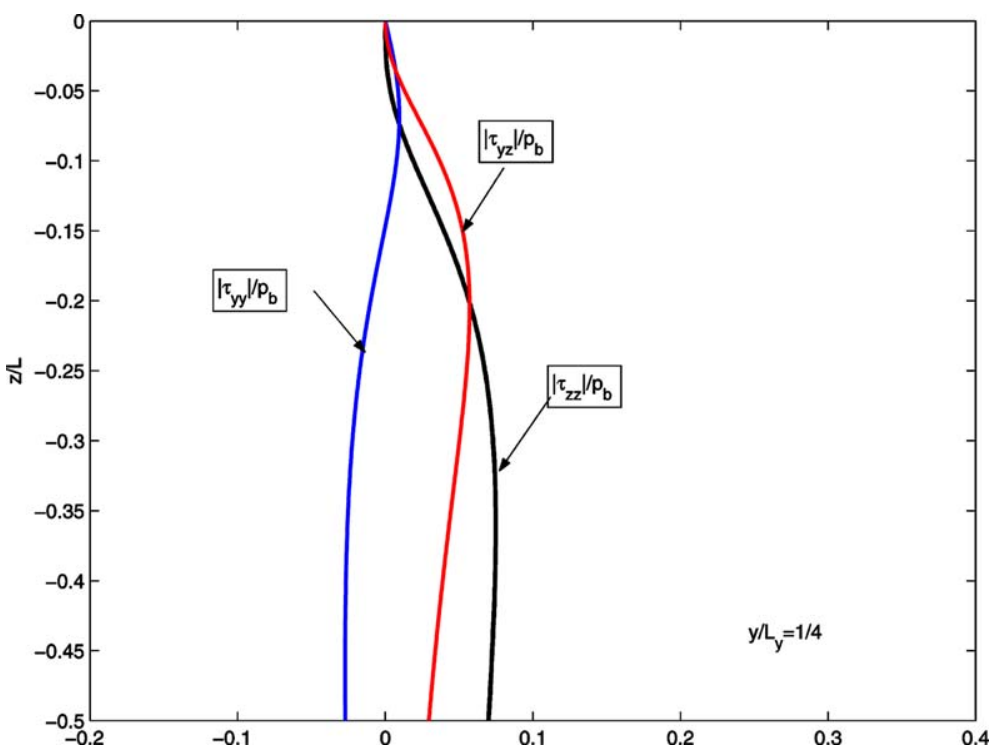

Fig. 18. Vertical distribution of added stresses, $\left|\tau_{y z}\right| / p_{\mathrm{b}}, \tau_{y y} / p_{\mathrm{b}}$ and $\left|\tau_{z z}\right| / p_{\mathrm{b}}$, due to self-weight of a breakwater at $y / L_{y}=1 / 4(s=20 \mathrm{~m})$. 


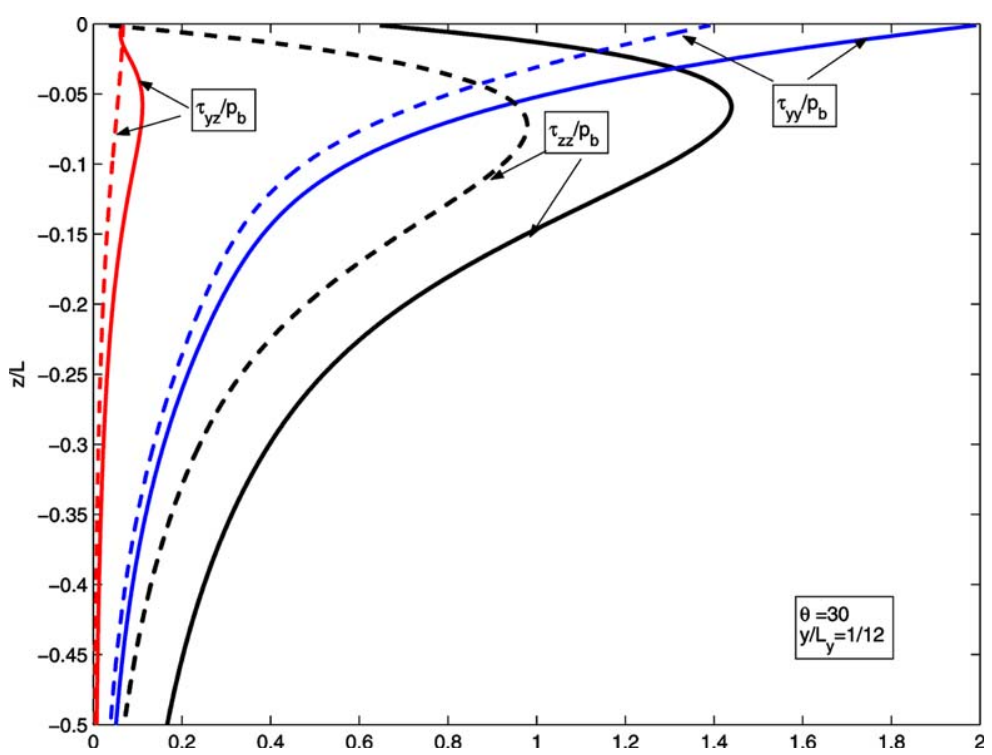

Fig. 19. Comparison of the stresses, $\left|\tau_{y z}\right| / p_{\mathrm{b}}, \tau_{y y} / p_{\mathrm{b}}$ and $\left|\tau_{z z}\right| / p_{\mathrm{b}}$, with and without consideration of the self-weight of a breakwater at $y / L_{y}=1 / 12(s=20 \mathrm{~m})$.

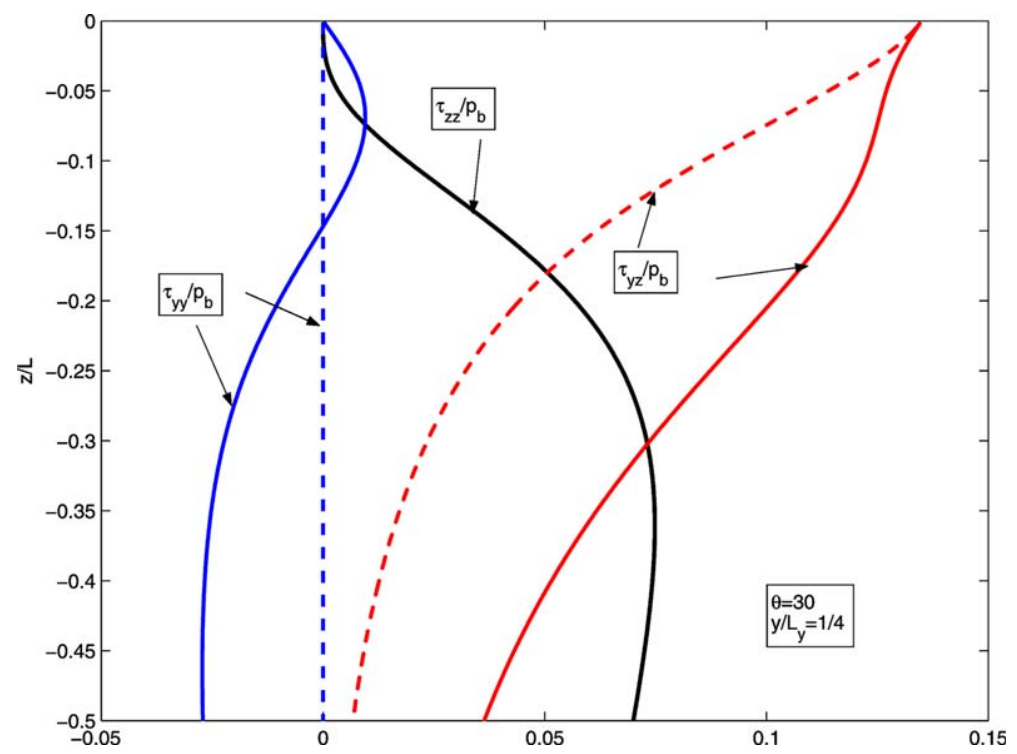

Fig. 20. Comparison of the stresses, $\left|\tau_{y z}\right| / p_{\mathrm{b}}, \tau_{y y} / p_{\mathrm{b}}$ and $\left|\tau_{z z}\right| / p_{\mathrm{b}}$, with and without consideration of the self-weight of a breakwater at $y / L_{y}=1 / 4(s=20 \mathrm{~m})$. 


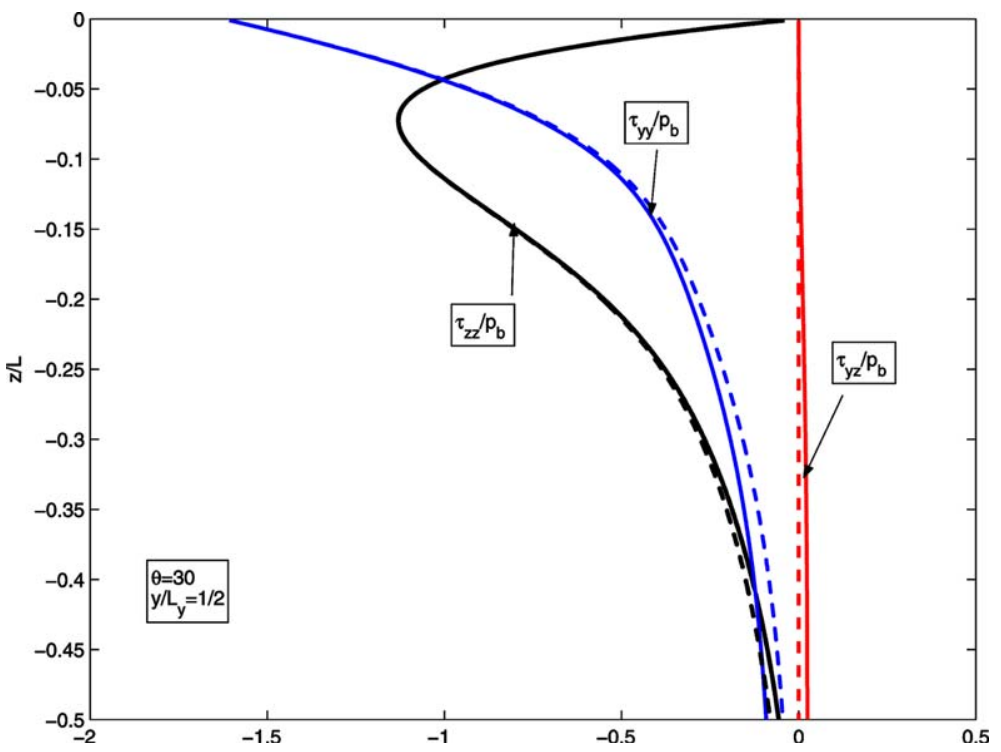

Fig. 21. Comparison of the stresses, $\left|\tau_{y z}\right| / p_{\mathrm{b}}, \tau_{y y} / p_{\mathrm{b}}$ and $\left|\tau_{z z}\right| / p_{\mathrm{b}}$, with and without consideration of the self-weight of a breakwater at $y / L_{y}=1 / 2(s=20 \mathrm{~m})$.

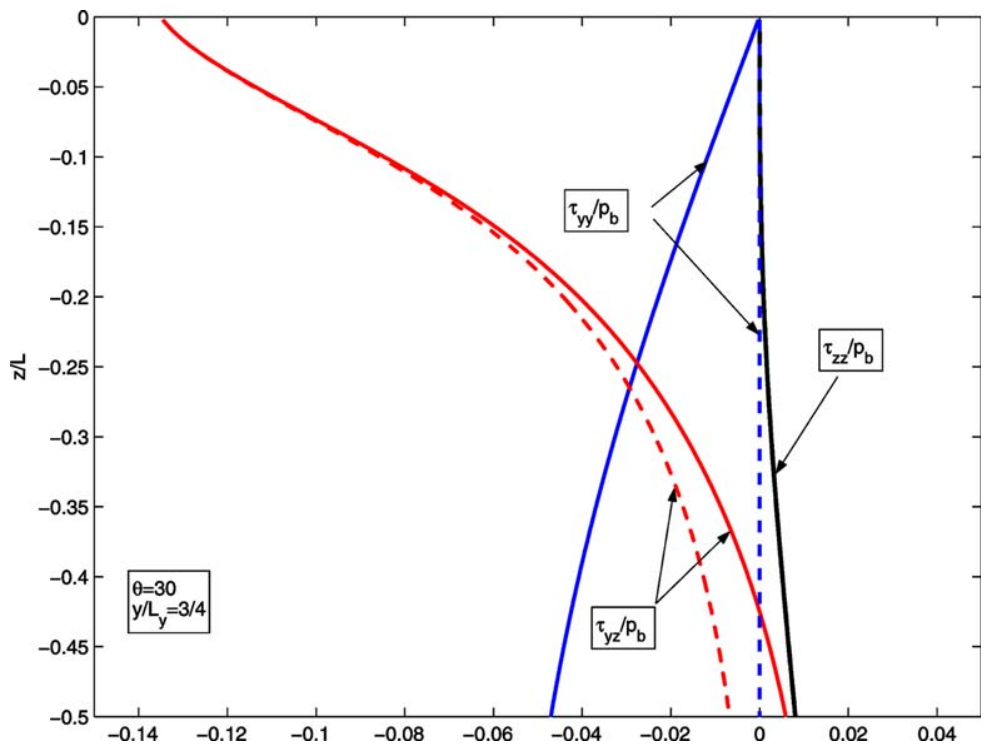

Fig. 22. Comparison of the stresses, $\left|\tau_{y z}\right| / p_{\mathrm{b}}, \tau_{y y} / p_{\mathrm{b}}$ and $\left|\tau_{z z}\right| / p_{\mathrm{b}}$, with and without consideration of the self-weight of a breakwater at $y / L_{y}=3 / 4(s=20 \mathrm{~m})$. 


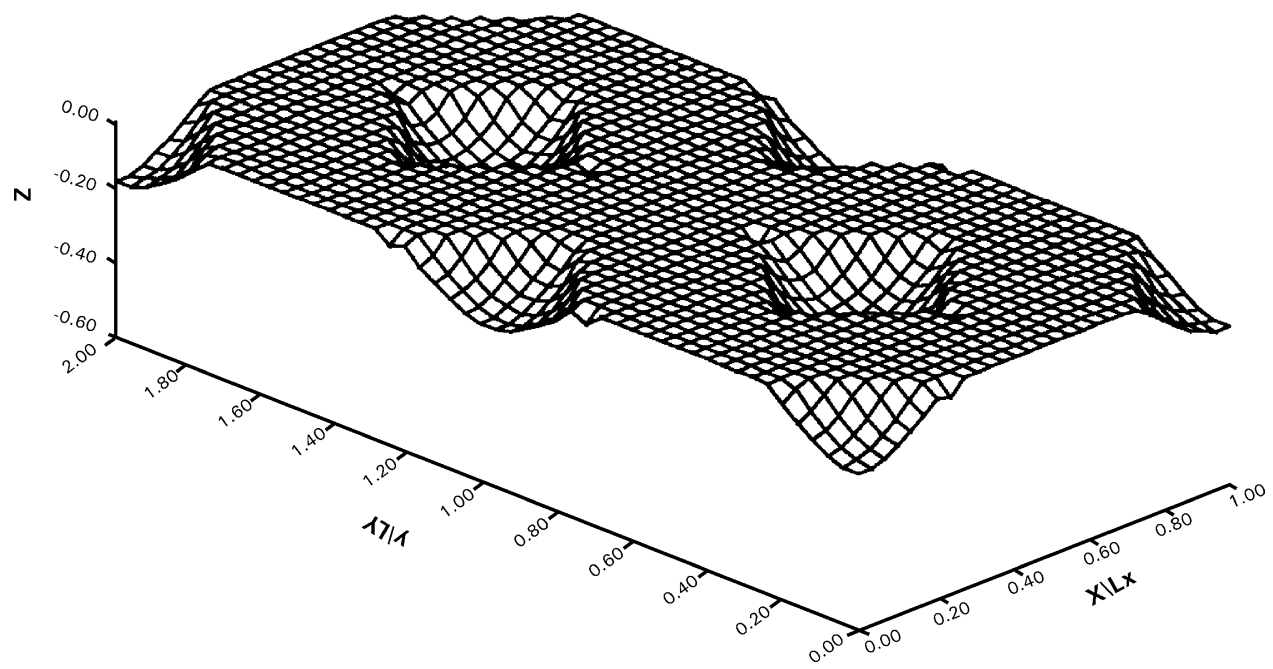

Fig. 23. Contour distribution of the wave-induced shear failure without self-weight of a breakwater.

Figs. 23 and 24, which show three-dimensional graphs for the wave-induced shear failure. Figs. 23 and 24 further show that inclusion of added stresses significantly affects the shear failure depth in the region of $0<y / L_{y}<1 / 4$ and $0<|z / L|<0.3$. Thus, in engineering practice, the consideration of self-weight of a breakwater is important in the above region.

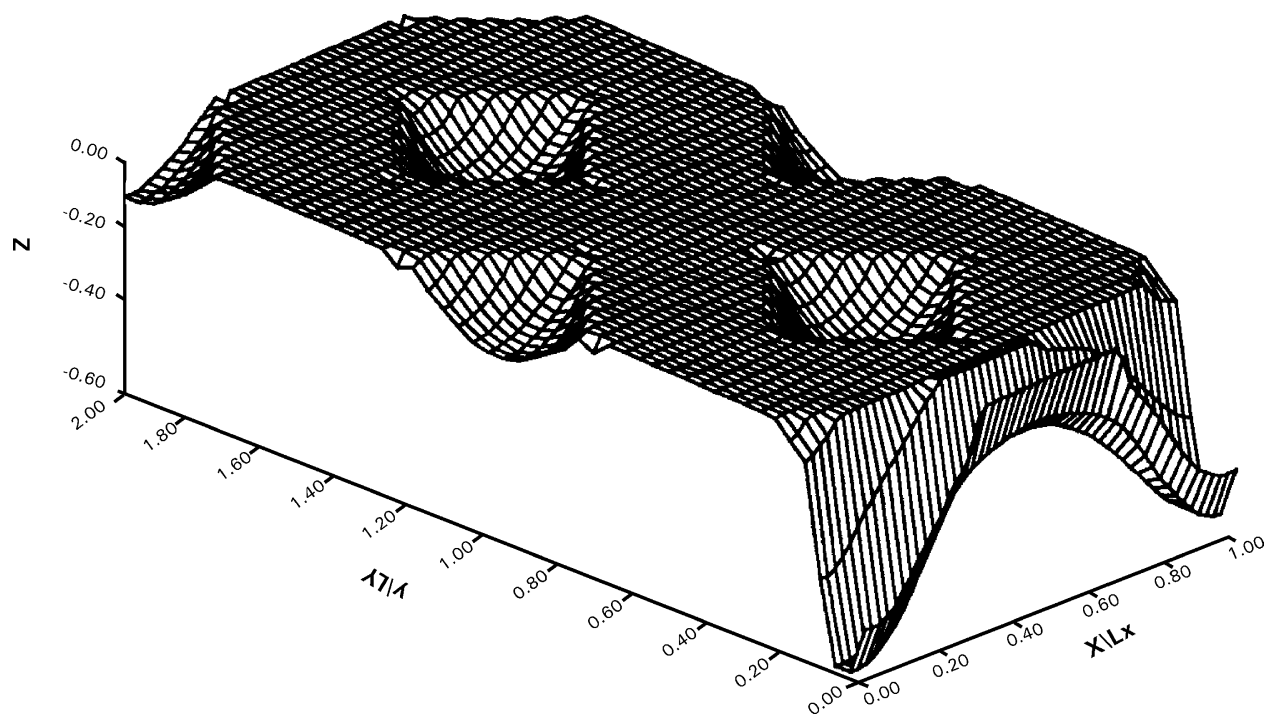

Fig. 24. Contour distribution of the wave-induced shear failure with self-weight of a breakwater. 


\section{Conclusions}

In this paper, a semi-analytical solution for the short-crested wave-induced liquefaction and shear failure in a Coulomb-damping porous seabed is proposed. In the model, partial reflection is considered. Based on numerical examples presented, the following conclusions can be drawn:

1. The liquefaction and shear failure depth induced by short-crested wave may not always vary between progressive and standing waves, especially at the surface of the seabed.

2. For coarse sand, the liquefaction depth does not vary with the incident wave angle, while the shear failure depth increases as the incident angle increases. The liquefaction and shear failure depths increases and wave period increases, but as relative water depth decreases.

3. For fine sand, incident wave angles significant affects the region of instability. In general, the liquefaction depth increases as incident wave angle increase, and the maximum occurs at $\theta=60^{\circ}$.

4. Generally speaking, the liquefaction and shear failure depths increase as reflection coefficient $K_{\mathrm{r}}$ increases in fine sand. The influence of incident wave angle on the shear failure is more significant than liquefaction depth.

5. Consideration of the added stresses due to self-weight of the breakwater is particularly important in the region of $0<y / L_{y}<1 / 4$ and $0<|z / L|<0.3$.

\section{Acknowledgements}

The authors thank for the financial support from NSFC funds \#40176027 (China) and ARC Large Grant \#A00104092 (Australia).

\section{References}

Biot, M.A., 1956. Theory of propagation of elastic waves in a fluid-saturated porous solid: I. Low frequency range. The Journal of Acoustical Society of America 28 (2), 168-178.

Carter, T.C., Liu, P.L.F., Mei, C.C., 1973. Mass transport by waves and offshore sand bedforms. Journal of Waterway, Harbour and Coastal Engineering Division, A.S.C.E. 99 (2), 165-184.

Fuchs, R.A., 1952. On the theory of short-crested oscillatory waves, Circular No 521, Gravity waves. US National Bureau of Standards, Gaithersburg, Md., pp. 187-200.

Gao, X.P., Zhao, Z.D., 1995. The scouring character and forecasting induced by standing waves in front of breakwaters. Acta Oceanological Sinica 17 (5), 140-146.

Griffith, D.V., 1986. Some theoretical observation on conical failure criteria in principal stress space. International Journal of Solids and Structures 22, 553-565.

Hsu, J.R.C., Silvester, R., Tsuchiya, Y., 1980. Boundary-layer velocities and mass transport in shortcrested waves. Journal of Fluid Mechanics 99, 321-342.

Hsu, J.R.C., Silvester, R., 1989. Mode test results of scouring along breakwater. Journal of Waterway, Port, Coastal and Ocean Engineering, A.S.C.E. 115 (1), 66-85.

Hsu, J.R.C., Tsuchiya, Y., Silvester, R., 1979. Third-order approximation to short-crested waves. Journal of Fluid Mechanics 90, 179-196.

Irie, I., Ndaoka, K., 1984. Laboratory reproduction of seabed scour in front of a breakwaters. In: Proceedings of 19th International Conference on Coastal Engineering, A.S.C.E., vol. 2, pp. 1715-1731. 
Jan, C.D., Lin, M.C., 1998. Bed forms generated on sandy bottom by oblique standing waves. Journal of Waterway, Port, Coastal, and Ocean Engineering, A. S. C. E. 124 (6), 295-302.

Jeng, D.-S., 1997. Wave-induced seabed response in front of a breakwater. PhD Thesis, The University of Western Australia, Australia, $297 \mathrm{pp}$.

Jeng, D.-S., 2002. Wave kinematics of partial reflection from a vertical wall. Ocean Engineering 29 (13), $1711-1724$.

Jeng, D.-S., Cha, J.R.C., 2003. Effects of dynamic soil behaviour and wave non-linearity on the waveinduced pore pressure and effective stresses in porous seabed. Ocean Engineering 30 (16), 2065-2089.

Jeng, D.-S., Lee, T.L., 2001. Dynamic response of porous seabed to ocean waves. Computers and Geotechnics 28, 99-128.

Jeng, D.-S., Rahman, J.R.C., Lee, T.L., 1999. Effects of inertial forces on wave-induced seabed response. International Journal of Offshore and Polar Engineering 9, 307-313.

Jeng, D.-S., Rahman, M.S., 2000. Effective stresses in a porous seabed of finite thickness: inertial effects. Canadian Geotechnical Journal 37 (4), 1383-1392.

Lee, T.L., Tsai, J.R.C., Jeng, D.-S., 2002. Ocean waves propagating over a Coulomb-damped poroelastic seabed of finite thickness. Computers and Geotechnics 29, 119-149.

Lin, M., 2001. The analysis of silt behaviors induced by water waves. Science in China (Series E) 44 (3), $239-250$.

Lin, M., 2003. Comparison the dynamic response of a saturated soil between the two models. Science in China (Series D) 33 (4), 392-400.

Lin, M., Jeng, D.-S., 2003. Comparison of existing poroelastic models for wave damping in a porous seabed. Ocean Engineering 30 (11), 1335-1352.

Lin, M.C., Wu, C.T., Lu, R.A., Liang, N.K., 1986. Effects of short-crested waves on the scouring around the breakwater. In: Proceedings of 20th International Conference on Coastal Engineering, A.S.C.E., pp. 2050-2064.

Silvester, R., 1972. Wave reflection at seawall and breakwaters. In: Proceedings of Institute of Civil Engineers, Landon, Part 2, vol. 51, pp. 123-131.

Sumer, M.B., Fredsoe, J., 2000. Experimental study of 2D scour and its protection at a rubble-mound breakwater. Coastal Engineering 40, 59-87.

Tanaka, N., Irie, I., Ozasa, H., 1972. A study on the velocity distribution of mass transport caused by diagonal partial standing waves. Report of the Port and Harbour Research Institution, 11(3), $112-137$.

Tsai, C.P., 1995. Wave-induced liquefaction potential in a porous seabed in front of a breakwater. Ocean Engineering 22 (1), 1-18.

Xie, S.L., 1983. Scouring patterns in front of the vertical breakwaters and their influences on the stability of the foundation of the breakwaters. Acta Oceanologica Sinica 5 (6), 808-823.

Yamamoto, T., 1982. On the response of a Coulomb-damped poroelastic bed to water waves. Marine Geotechnology 5 (2), 93-127.

Yamamoto, T., Koning, H.Y., Sellmeijer, H., van Hijum, E., 1978. On the response of a poro-elastic bed to water waves. Journal of Fluid Mechanics 87, 193-206.

Zen, K., Yamazaki, H., 1993. Wave-induced liquefaction in a permeable seabed. Report of the Port and Harbour Research Institution, Japan 31, 155-192. 